\title{
JImproved Prediction of Cold-Air Pools in the Weather Research and Forecasting Model Using a Truly Horizontal Diffusion Scheme for Potential Temperature
}

\author{
Robert S. ARTHUR, ${ }^{a}$ KATHERINE A. Lundquist, ${ }^{a}$ AND JosePh B. OLSON ${ }^{b}$ \\ ${ }^{\text {a }}$ Lawrence Livermore National Laboratory, Livermore, California \\ ${ }^{\mathrm{b}}$ Global Systems Laboratory, National Oceanic and Atmospheric Administration, Boulder, Colorado
}

(Manuscript received 17 July 2020, in final form 21 October 2020)

\begin{abstract}
The terrain-following vertical coordinate system used by many atmospheric models, including the Weather Research and Forecasting (WRF) Model, is prone to errors in regions of complex terrain. These errors stem, in part, from the calculation of horizontal gradients within the diffusion term of the momentum or scalar evolution equations. In WRF, such gradients can be calculated along coordinate surfaces, or using metric terms that help account for grid skewness. However, neither of these options ensures a truly horizontal gradient calculation, especially if a grid cell is skewed enough that the heights of the neighboring grid points used in the calculation fall outside the vertical range of the cell. In this work, an improved scheme that uses Taylor series approximations to vertically interpolate variables to the level necessary for a truly horizontal gradient calculation is implemented in WRF for the diffusion of potential temperature. The scheme is validated using an atmosphere-at-rest configuration, in which spurious flows develop only as a result of numerical errors and can thus be used as a proxy for model performance. Following validation, the method is applied to the simulation of cold-air pools (CAPs), which occur in regions of complex terrain and are characterized by strong near-surface temperature gradients. Using the truly horizontal scheme, idealized simulations demonstrate reduced numerical mixing in a quiescent CAP, and a realistic case study in the Columbia River basin shows a reduction in positive wind speed bias by up to roughly $20 \%$ compared to observations from the Second Wind Forecast Improvement Project.
\end{abstract}

KEYWORDS: Cold pools; Mixing; Model errors; Numerical weather prediction/forecasting

\section{Introduction}

\section{a. Background and motivation}

Mesoscale atmospheric models, such as the Weather Research and Forecasting (WRF) Model (Skamarock et al. 2019), commonly use a terrain-following vertical coordinate system (Gal-Chen and Somerville 1975). These coordinates have the advantage of aligning the terrain with the lower coordinate surface, making the implementation of surface boundary conditions straightforward. One drawback of terrain-following coordinates, however, is that they can become highly skewed over steep or complex terrain. If not accounted for properly, grid skewness leads to substantial numerical errors stemming from the calculation of horizontal gradients (Janjić 1977, 1989).

Historically, mesoscale models have been used at relatively coarse resolutions (with horizontal grid spacings on the order of $10 \mathrm{~km}$ ), where terrain is coarsely sampled, and therefore slopes appear relatively gentle. As mesoscale models are used at finer resolution, additional terrain detail is resolved, resulting in steeper terrain slopes that increase errors related to grid skewness. For example, the operational High-Resolution Rapid Refresh model (HRRR; Benjamin et al. 2016), which covers the continental United States with $3 \mathrm{~km}$ horizontal grid spacing, has a maximum slope of roughly $26^{\circ}$.

๖ Denotes content that is immediately available upon publication as open access.

Corresponding author: Robert S. Arthur, arthur7@llnl.gov
Several efforts have been undertaken in the literature to reduce numerical errors related to terrain-following coordinates in atmospheric models. A common approach is to use a hybrid coordinate, which flattens with height more quickly than a standard terrain-following coordinate, thus reducing grid skewness away from the terrain (Arakawa and Lamb 1977; Simmons and Burridge 1981; Schär et al. 2002; Klemp 2011). A "hybrid sigma-pressure" coordinate was implemented in WRF following Park et al. (2013) in version 3.9, and this became the default coordinate option in version 4 (used herein, see Skamarock et al. 2019).

Even with a hybrid coordinate, however, grid skewness remains over steep topography, especially near the surface. Therefore, improved finite difference schemes that better account for grid skewness can further reduce model error. Mahrer (1984) proposed a scheme based on an alternate numerical stencil that minimizes horizontal skewness, thus calculating "truly horizontal" gradients. Subsequent studies have applied similar methods in different models (Dempsey and Davis 1998; Klemp 2011; Zängl 2002, 2012). In this work, a truly horizontal finite difference scheme similar to that of Mahrer (1984) is implemented in the WRF Model for the diffusion of potential temperature. While a similar scheme could be applied to other model variables, the present implementation focuses on potential temperature as a proof of concept within the WRF framework that has immediate application to cold-air pool (CAP) forecasting, as discussed below.

The implementation is first validated using an atmosphereat-rest test case, which is commonly used in the literature for this purpose (e.g., Dempsey and Davis 1998; Klemp 2011; 
Zängl 2012). Such cases are initialized with a quiescent atmosphere that includes a stable temperature sounding with discontinuities (e.g., inversion layers). The model is then run for a period of time in the absence of forcing, such that in the analytical solution, no perturbations would develop. However, in the model, spurious perturbations and flows develop due to numerical errors, and the magnitude of these can be used to assess the accuracy of the numerical method.

\section{b. Case study selection}

Following validation of the truly horizontal potential temperature diffusion scheme in WRF, the method is applied to CAP simulations. CAPs are near-surface stable layers that form within valleys or basins, usually in the nighttime or wintertime, and can persist on time scales from hours to days (see review of Zardi and Whiteman 2013). Interest in CAPs stems from their tendency to trap pollution in urban areas (e.g., Silcox et al. 2012; Lareau et al. 2013; Whiteman et al. 2014), and, more recently, from their effect on wind energy production (e.g., McCaffrey et al. 2019; Pichugina et al. 2019). CAP events also pose risks to air and ground transportation, as well as agricultural activities, due to an increased likelihood of low clouds, fog, or freezing rain (as noted by Lareau et al. 2013; Sheridan 2019).

A variety of observational (e.g., Whiteman et al. 2008; Lareau et al. 2013; Lehner et al. 2016) and modeling (e.g., Zhong et al. 2001; Billings et al. 2006; Vosper and Brown 2008; Lu and Zhong 2014; Lareau and Horel 2015; Sheridan 2019) studies have examined the dynamics of CAPs, including their formation, persistence, and ultimate erosion. Indeed, Billings et al. (2006) demonstrated model improvement for a CAP case using the truly horizontal diffusion method of Zängl (2002) within the fifth-generation Pennsylvania State UniversityNational Center for Atmospheric Research (PSU-NCAR) Mesoscale Model (MM5; Grell et al. 1994). However, CAPs remain a forecasting challenge (see, e.g., Mahrt 1998) due to their relatively small-scale dynamics, including stratified layers and near-surface slope flows. Commonly used coarse model configurations are often not able to fully resolve these features. Adding to this challenge is the strong dependence of CAP dynamics on parameterized model physics, such as radiation and surface fluxes.

Because CAPs generally occur in regions of steep, complex terrain, they provide a useful test case for the truly horizontal potential temperature diffusion scheme implemented here. Specifically, numerical mixing induced by the horizontal differencing scheme in regions of steep terrain can reduce the predicted persistence of CAPs. Thus, as a test of the present implementation, two CAP cases are completed in WRF. The first is an idealized extension of the atmosphere-at-rest validation case that demonstrates the effect of spurious numerical mixing on CAP persistence. The second is a realistic case from the Second Wind Forecast Improvement Project (WFIP2; Shaw et al. 2019), where CAPs were noted as one of the greatest challenges for wind energy forecasting in the complex terrain of the Columbia River basin (Wilczak et al. 2019).

The WFIP2 project consisted of an 18-month observation campaign in the northwest United States (Wilczak et al. 2019), as well as an extensive effort to improve mesoscale model capabilities for wind energy forecasting (Olson et al. 2019). Model development was focused on the aforementioned HRRR model, which has $3 \mathrm{~km}$ horizontal grid spacing and uses the Advanced Research version of WRF. A special "provisional" setup of the HRRR was employed during WFIP2, including a $3 \mathrm{~km}$ WFIP2 HRRR domain over the western United States and a $750 \mathrm{~m}$ WFIP2 HRRRNEST over the northwest United States, centered on the Columbia River basin (see Fig. 8 in section 4). The WFIP2 HRRR/HRRRNEST setup employs the same physics suite as the operational HRRR model, but is run in a nonoperational "cold-start" configuration, as detailed in Olson et al. (2019). The realistic cold pool simulation presented here follows the WFIP2 HRRR/HRRRNEST setup, and several WFIP2 observation datasets are utilized for model comparison. As discussed in Shaw et al. (2019), both the WFIP2 HRRR/HRRRNEST setup files and the observation datasets are publicly available through the U.S. Department of Energy Data Archive and Portal (DAP; http://a2e.energy.gov/data).

\section{Calculation of horizontal diffusion}

\section{a. Standard options in WRF}

In the WRF potential temperature tendency equation,

$$
\frac{\partial \theta}{\partial t}=\cdots+\frac{\partial H_{x}}{\partial x}
$$

horizontal gradients of $H_{x}$, which represents the parameterized horizontal flux of heat due to unresolved turbulence, must be calculated in a discrete sense. The calculation of $H_{x}$ itself depends on the horizontal gradient in potential temperature,

$$
H_{x}=K_{H}^{h} \frac{\partial \theta}{\partial x},
$$

with $K_{H}^{h}$ as the horizontal eddy diffusion coefficient for heat. Equations (1) and (2), and those that follow are presented in two dimensions $(x-z)$ for simplicity, although analogous terms representing horizontal diffusion in the $y$ direction are included in the WRF Model. Note that some terms (e.g., nondiffusion terms) and variables in the WRF Model equations (e.g., map scale factors and column mass, see Skamarock et al. 2019) are not presented here, but are included in the model implementation. Averaging operations that account for WRF's staggered grid are included in the present discussion only as necessary. In the discretized equations below, the indices $i$ and $k$ represent horizontal and vertical grid locations, respectively, on WRF's Arakawa C staggered grid. Thus, for a given grid cell, $i(k)$ represents the left (bottom) cell face and cell center, while $i+1(k+1)$ represents the right (top) cell face. The potential temperature $\theta$ is defined at the cell center, while the horizontal diffusive flux $H_{x}$ is defined on cell faces (see Fig. 1).

The standard WRF package has two available options for calculating horizontal gradients within the diffusion operator. The most straightforward option (activated by the namelist option diff_opt $=1$ ) is to calculate gradients along coordinate surfaces, as in Fig. 1a. Thus, Eq. (2) is discretized as 


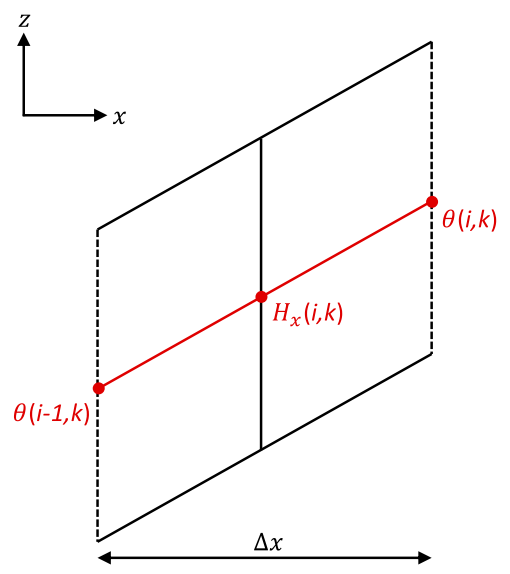

(a) Along coordinate surfaces WRF diff_opt $=1$

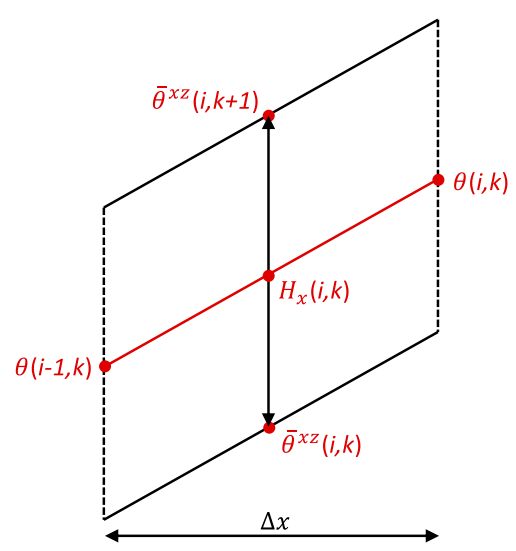

(b) In physical space WRF diff opt $=2$

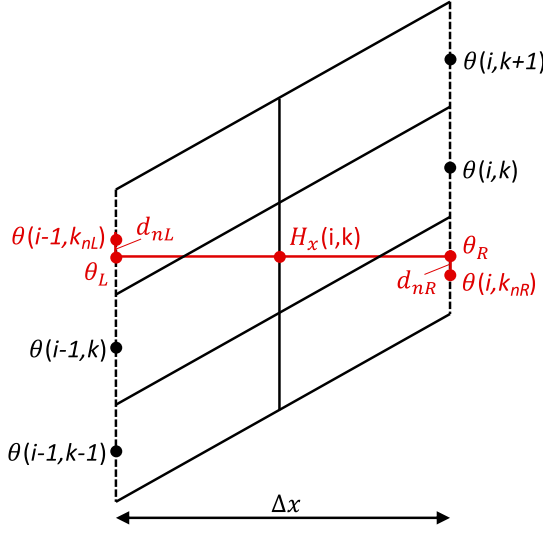

(c) Truly horizontal Present implementation

FIG. 1. Two-dimensional $x-z$ illustration of horizontal gradient calculation options for the diffusion operator in WRF. Solid black lines denote cell faces, while dashed black lines denote cell centers. The horizontal index $i$ and the vertical index $k$ represent locations on WRF's staggered grid, as explained in the text. Values used in the gradient calculation are highlighted in red. (c) $k_{n L}=k+1$ and $k_{n R}=k-1$ denote the grid levels from which a vertical Taylor series approximation is applied in order to calculate $\theta_{L}$ and $\theta_{R}$, respectively. Calculations are shown for the diffusive flux in the $x$ direction $H_{x}$. Similar calculations are made for $H_{y}, \partial H_{x} / \partial x$, and $\partial H_{y} / \partial y$, but at different locations on WRF's staggered grid.

$$
H_{x}(i, k)=K_{H}^{h}\left[\frac{\theta(i, k)-\theta(i-1, k)}{\Delta x}\right]
$$

and similarly for the right-hand side of Eq. (1). In cases with relatively flat topography, such as mesoscale simulations with coarsely resolved terrain features, this method closely approximates the desired horizontal gradient. However, since coordinate surfaces in WRF generally follow the terrain, either throughout the vertical extent of the domain with the original terrain-following sigma coordinate, or near the surface with the new hybrid sigma-pressure coordinate, errors are introduced when diff_opt $=1$ is used over steeper terrain slopes.

For this reason, WRF also includes an option to calculate horizontal gradients within the diffusion scheme in physical space, as in Fig. 1b. This option (activated by the namelist option diff_opt $=2$ ) introduces metric terms to help account for grid skewness. The diffusion term in Eq. (1) thus becomes

$$
\frac{\partial H_{x}}{\partial x}-z_{x} \frac{\partial H_{x}}{\partial z}
$$

and Eq. (2) becomes

$$
H_{x}=K_{H}^{h}\left(\frac{\partial \theta}{\partial x}-z_{x} \frac{\partial \theta}{\partial z}\right)
$$

The metric term is defined as $z_{x}=\partial z / \partial x$. Equation (5) is discretized as

$H_{x}(i, k)=K_{H}^{h}\left[\frac{\theta(i, k)-\theta(i-1, k)}{\Delta x}-\frac{\Delta z_{\mathrm{def}}}{\Delta x} \frac{\bar{\theta}^{x z}(i, k+1)-\bar{\theta}^{x z}(i, k)}{\Delta z}\right]$,

and similarly for Eq. (4). Here, $\bar{\theta}^{x z}$ denotes $\theta$ values that have been averaged spatially in the $x$ and $z$ directions (see Fig. 1b).
Note that $\Delta z_{\mathrm{def}}=z_{\theta}(i, k)-z_{\theta}(i-1, k)$ represents the local vertical grid deformation, while $\Delta z=z_{\bar{\theta}^{x z}}(i, k+1)-z_{\bar{\theta}^{x z}}(i, k)$ is the local vertical grid spacing $\left(z_{\theta}\right.$ and $z_{\bar{\theta}} x z$ are the vertical positions of $\theta$ and $\bar{\theta}^{x z}$ points, respectively).

The use of diff_opt $=2$ can improve the accuracy of gradient calculations in WRF over sloped terrain. However, performance is limited by the implementation because the metric terms are calculated using neighboring points on the top and bottom of the grid cell only, as illustrated in Fig. 1b. Thus, if the grid cell is skewed enough such that $\Delta z_{\text {def }}>\Delta z$, as in Fig. 1c, errors will still occur in the calculation of horizontal derivatives.

\section{b. Truly horizontal diffusion calculation}

An improved method would take advantage of additional, nonadjacent grid points in order to calculate a more accurate horizontal gradient. Herein, such a method is implemented following Mahrer (1984). Specifically, the WRF code that calculates the potential temperature tendency due to horizontal diffusion when diff_opt $=2$ is modified to identify the vertical $k$ index for neighboring points that minimizes vertical distance in the stencil. Then, Taylor series approximations are used in the vertical direction to calculate the variable value at the vertical level at which the horizontal gradient is required (see $\theta_{L}$ and $\theta_{R}$ in Fig. $1 \mathrm{c}$ ).

To accomplish this, a search is performed on either side of the gradient to determine the nearest vertical grid level, denoted $k_{n L}$ and $k_{n R}$, respectively, from which to apply a vertical Taylor series approximation. The horizontal gradient $H_{x}$ is then discretized as

$$
H_{x}(i, k)=K_{H}^{h}\left[\frac{\theta_{R}-\theta_{L}}{\Delta x}\right]
$$



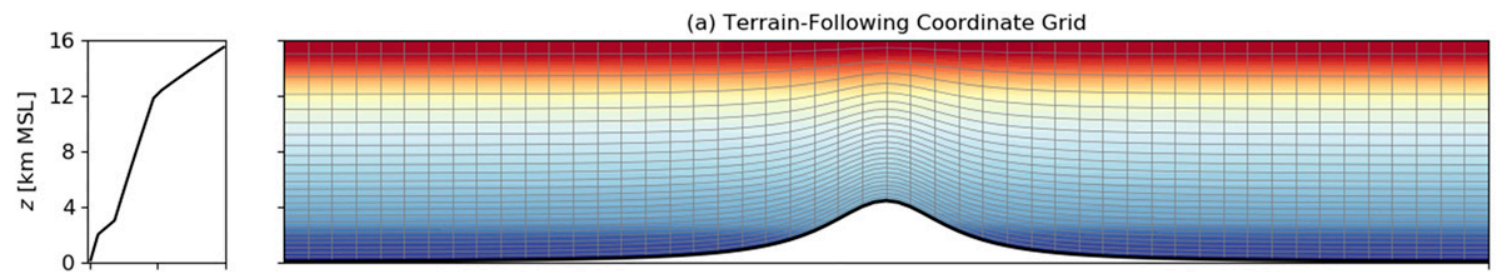

(b) Hybrid Coordinate Grid
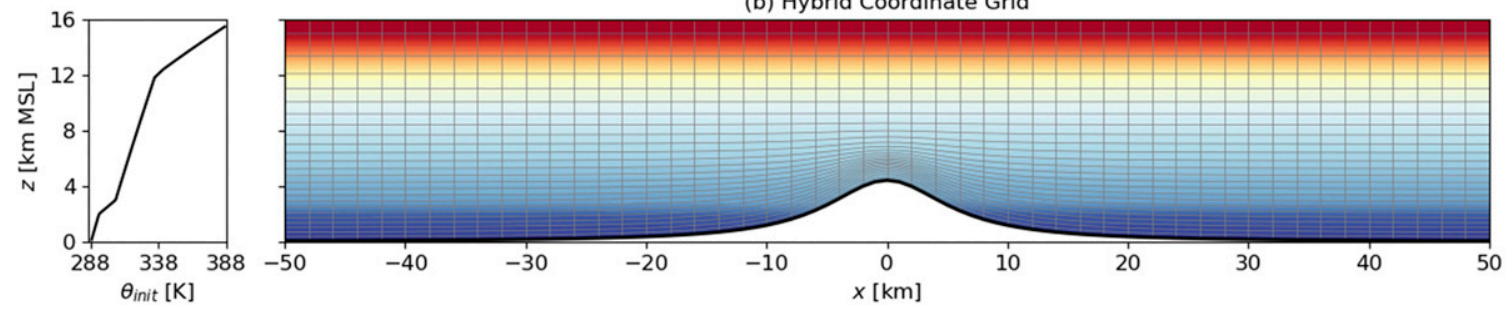

FIG. 2. (right) Two-dimensional $x-z$ slices of the (a) terrain-following and (b) hybrid coordinate grid options used in standard atmosphereat-rest test cases, with every other grid line shown for clarity. (left) Initial potential temperature field $\theta_{\text {init }}$.

where

$$
\begin{aligned}
& \theta_{L}=\theta\left(i-1, k_{n L}\right)+\left.d_{n L} \frac{\partial \theta}{\partial z}\right|_{\left(i-1, k_{n L}\right)}, \\
& \theta_{R}=\theta\left(i, k_{n R}\right)+\left.d_{n R} \frac{\partial \theta}{\partial z}\right|_{\left(i, k_{n R}\right)} .
\end{aligned}
$$

This requires the calculation of the vertical gradient term in the Taylor series, $\partial \theta / \partial z$, as well as the quantities $d_{n L}$ and $d_{n R}$, which represent the (signed) vertical distances between $H_{x}(i$, $k$ ) and $\theta\left(i-1, k_{n L}\right)$ and $\theta\left(i, k_{n R}\right)$, respectively. It should be noted that this calculation essentially reverts to the standard diff_opt $=2$ formulation if the closest vertical indices identified on the left and right sides are equal to the index for which the derivative is being calculated (i.e., $k_{n L}=k_{n R}=k$ ).

When the new method is used, care must be taken near the bottom and top boundaries, where the appropriate level for a truly horizontal gradient may be outside of the atmospheric domain. This is most important near the surface, where boundary layer dynamics are of interest and the grid is generally the most skewed. In the present implementation, if a truly horizontal gradient would require a quantity beneath the terrain surface, the horizontal gradient is instead extrapolated downward from the lowest vertical level at which a quantity beneath the terrain surface is not necessary. A linear extrapolation routine that utilizes the first two available model levels is employed. Other techniques, such as extrapolating quantities downward and then calculating the gradient (as opposed to extrapolating the gradient itself), or taking a one-sided difference, were attempted with less success. Since the present surface implementation is sufficient for the cases tested herein, the use of other extrapolation techniques or higher-order methods is left for future work. At the model top, which is relatively flat, no extrapolation is performed; instead, the top vertical level is used in the Taylor series approximation.

The process described above for a truly horizontal gradient calculation is completed similarly for each gradient needed within the potential temperature diffusion scheme (i.e., $H_{x}, H_{y}$, $\partial H_{x} / \partial x$, and $\left.\partial H_{y} / \partial y\right)$. As is standard in WRF, $K_{H}=K_{M} / \mathrm{Pr}$, where $K_{M}$ is the eddy diffusion coefficient for momentum and $\operatorname{Pr}=1 / 3$ is the Prandtl number. Horizontal diffusion of prognostic variables other than the potential temperature is calculated as with diff_opt $=2$. Because the calculation of $K_{M}$ is based on velocity gradients, this implies that the value of $K_{H}$ used for potential temperature diffusion is based on the standard diff_opt $=2$ formulation rather than the truly horizontal scheme. To mitigate this complication, constant values of $K_{M}$ and $K_{H}$ are used in the idealized cases (though not the real case) presented below. Despite this caveat, the present truly horizontal potential temperature diffusion scheme is compatible with all standard WRF options for calculating the eddy diffusion coefficients $\left(\mathrm{km} \_\mathrm{opt}=1-4\right)$. The calculation of $K_{M}$, and by extension $K_{H}$, could be updated in the future to employ the truly horizontal gradient formulation, although preliminary attempts showed little or no gain in model performance, and are therefore not presented here.

\section{Validation of truly horizontal potential temperature diffusion scheme}

\section{a. Standard atmosphere-at-rest case}

\section{1) Model Setup}

The truly horizontal potential temperature diffusion implementation is first validated using a standard atmosphere-at-rest test setup. Each case is run in the ideal mode of WRF v4.1.2 for 24 h over Agnesi hill topography,

$$
h(x, y)=h_{p}\left(1+\frac{x^{2}+y^{2}}{L^{2}}\right)^{-1},
$$

where $h_{p}=4.5 \mathrm{~km}$ is the peak elevation and $L=6 \mathrm{~km}$ is the horizontal length scale (see Fig. 2).

The domain covers $100 \mathrm{~km}^{2}$ with a horizontal grid spacing of $\Delta x=\Delta y=1 \mathrm{~km}$. This results in a maximum terrain slope of roughly $25^{\circ}$, similar to the maximum slope of $26^{\circ}$ in the standard HRRR domain. 
TABLE 1. Maximum absolute value of potential temperature perturbation $\theta-\theta_{\text {init }}(\mathrm{K})$ and velocity components $u$ and $w$ (m s ${ }^{-1}$ ) within the model domain after $24 \mathrm{~h}$ of simulation time for standard atmosphere-at-rest test cases. T-F indicates terrain-following.

\begin{tabular}{|c|c|c|c|c|c|}
\hline & Coordinate & Diffusion & $\left|\theta-\theta_{\text {init }}\right|$ & $|u|$ & $|w|$ \\
\hline \multirow[t]{6}{*}{ Large diffusivity } & $\mathrm{T}-\mathrm{F}$ & diff_opt $=1$ & 2.45 & 2.15 & 0.91 \\
\hline & Hybrid & diff_opt $=1$ & 2.31 & 1.99 & 0.87 \\
\hline & $\mathrm{T}-\mathrm{F}$ & diff_opt $=2$ & 0.31 & 1.58 & 1.53 \\
\hline & Hybrid & diff_opt $=2$ & 0.31 & 1.55 & 1.46 \\
\hline & $\mathrm{T}-\mathrm{F}$ & Truly horizontal & 0.31 & 0.82 & 0.27 \\
\hline & Hybrid & Truly horizontal & 0.27 & 0.88 & 0.30 \\
\hline \multirow[t]{6}{*}{ Small diffusivity } & $\mathrm{T}-\mathrm{F}$ & diff_opt $=1$ & 0.12 & 0.40 & 0.10 \\
\hline & Hybrid & diff_opt $=1$ & 0.08 & 0.48 & 0.08 \\
\hline & $\mathrm{T}-\mathrm{F}$ & diff_opt $=2$ & 0.09 & 0.15 & 0.02 \\
\hline & Hybrid & diff_opt $=2$ & 0.02 & 0.10 & 0.02 \\
\hline & $\mathrm{T}-\mathrm{F}$ & Truly horizontal & 0.09 & 0.14 & 0.02 \\
\hline & Hybrid & Truly horizontal & 0.01 & 0.09 & 0.02 \\
\hline
\end{tabular}

To study errors in calculating the horizontal gradient of potential temperature, the horizontal diffusion coefficient for heat $K_{H}^{h}$ is set to a constant and the vertical diffusion coefficient for heat $K_{H}^{v}$ is set to zero, as are both the horizontal and vertical diffusion coefficients for momentum, $K_{M}^{h}$ and $K_{M}^{v}$. This is accomplished using the WRF namelist option km_opt $=1$. Typically, when this option is used, the user sets $K_{M}^{h}$ and $K_{M}^{v}$ and WRF calculates $K_{H}=K_{M} / \mathrm{Pr}$. However, the WRF code was modified for these tests so that each $K$ value can be set independently.

We set $K_{H}^{h}$ to a value of either $1000 \mathrm{~m}^{2} \mathrm{~s}^{-1}$ (referred to as the large diffusivity case) or $10 \mathrm{~m}^{2} \mathrm{~s}^{-1}$ (referred to as the small diffusivity case). It is important to note that the atmosphere-atrest tests are highly idealized, and are designed primarily to highlight numerical errors in the horizontal potential temperature diffusion scheme. Thus, they do not represent realistic atmospheric conditions. Two gradually more realistic cases will be explored below in section $3 \mathrm{~b}$ and 4 .

Although validation is focused on the horizontal diffusion scheme, the definition of the vertical coordinate and the vertical grid spacing are important because they are factors in determining grid skewness, and thus how many vertical levels must be crossed in order to achieve a truly horizontal gradient calculation. The vertical grid used here has 49 levels (50 interface levels), and two vertical coordinate options are employed. First is WRF's original terrain-following sigma coordinate, which was the default for version 3 (Skamarock et al. 2008), and is set in version 4 using the namelist option hybrid_opt $=0$. Second is the hybrid sigma-pressure coordinate option, which is the default in version 4 and is set using hybrid_opt $=2$. The hybrid coordinate is a terrain-following sigma coordinate near the surface, but flattens out faster with altitude as it becomes a pure pressure-based coordinate (see comparison in Fig. 2). This option was introduced to mitigate errors related to the terrain-following sigma coordinate, especially far above the surface (Beck et al. 2020). It is therefore useful to investigate in combination with the truly horizontal diffusion scheme presented here.

In all, 12 simulations are completed using three methods of calculating the diffusion of potential temperature (diff_opt $=$ 1 , diff_opt $=2$, and truly horizontal), two vertical coordinate options (terrain-following and hybrid), and two diffusivity values (large and small); see Table 1. Each case has periodic lateral boundary conditions, and all atmospheric physics modules are turned off. The initial velocity is zero and the atmosphere is dry. The initial potential temperature profile $\theta_{\text {init }}(z)$ shown in Fig. 2, is defined using a Brunt-Väisälä frequency of $N=0.01 \mathrm{~s}^{-1}$ below the tropopause (at an altitude of $12 \mathrm{~km}$ ), and $N=0.02 \mathrm{~s}^{-1}$ above the tropopause. Additionally, there is an inversion layer of increased stability between 2 and $3 \mathrm{~km}$, also with $N=0.02 \mathrm{~s}^{-1}$. The inversion layer intersects the hill topography such that errors associated with the finite difference scheme are concentrated in this region.

Note that the atmosphere-at-rest test cases utilize the WRF namelist option mix_full_fields, which applies only to ideal cases. When mix_full_fields is used, the diffusion operator is applied to full prognostic variable fields, rather than a perturbation field that is defined relative to the initial condition. This option is available only for diff_opt $=2$ (not diff_opt $=1)$ in the standard WRF code. Thus, in order to make one-to-one comparisons in the present validation, a mix_full_fields option was implemented for diff_opt $=1$.

\section{2) RESults}

In the atmosphere-at-rest test setup, the potential temperature field should theoretically remain fixed in time. However, errors associated with the horizontal potential temperature gradient calculation cause spurious perturbations to develop, as shown in Fig. 3. This figure depicts the potential temperature perturbation for the large diffusivity case, for each diffusion and vertical coordinate option, after $24 \mathrm{~h}$ of simulation time. Perturbations as large as nearly $2.5 \mathrm{~K}$ develop after $24 \mathrm{~h}$ of simulation time when diff_opt $=1$ is used, but these are reduced to roughly $0.3 \mathrm{~K}$ for diff_opt $=2$ and the truly horizontal scheme. The errors in the potential temperature field lead to spurious flows, shown in the velocity fields in Fig. 4.

For each case in Figs. 3 and 4, an $x-z$ cross section is shown through the $y$ centerline of the domain (first and third rows of Figs. 3 and 4; cross section location denoted by dotted lines in the second and fourth rows), and a horizontal $x-y$ cross section is shown at a particular vertical level. 

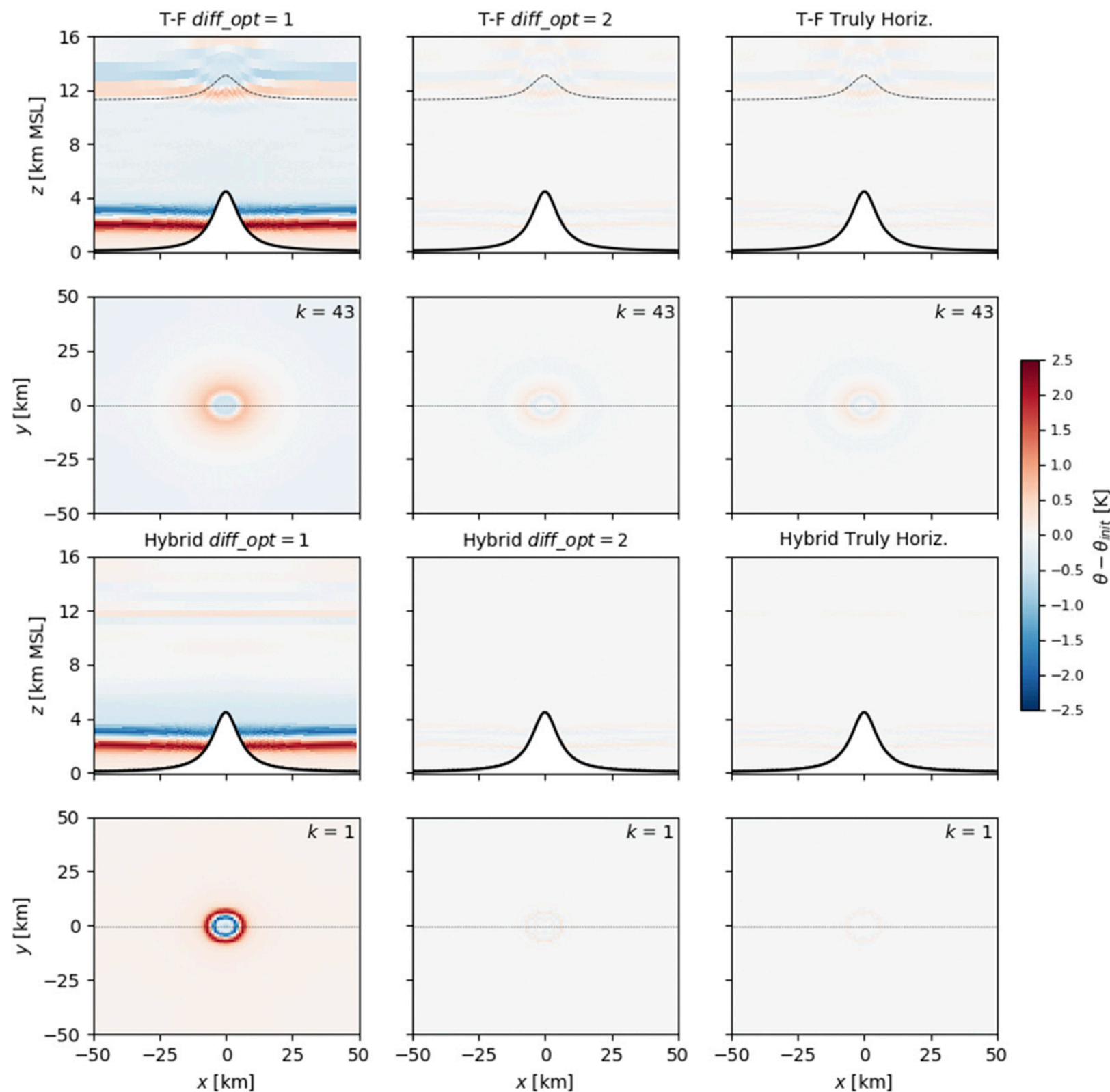

FIG. 3. Potential temperature perturbation $\theta-\theta_{\text {init }}$ after $24 \mathrm{~h}$ of simulation time for standard atmosphere-at-rest test cases with large diffusivity. Results are shown for (left) diff_opt $=1$, (center) diff_opt $=2$, and (right) the present truly horizontal implementation; for (top two rows) WRF's traditional terrain-following coordinate and (bottom two rows) the hybrid coordinate. For each case, an $x-z$ cross section is shown through the $y$ centerline of the domain (first and third rows), and an $x-y$ cross section is shown along a specific vertical level (second and fourth rows). Dotted lines depict cross-section locations in corresponding plots. For terrain-following cases, the $x-y$ cross section is shown at $k=43$ (denoted by the dotted line in the first row), while for hybrid cases, it is shown at the first vertical level, $k=1$ (dotted line in third row not visible).

For terrain-following coordinate cases, the cross section is taken along grid level $k=43$, which intersects the tropopause (second row of Figs. 3 and 4; cross section location denoted by dotted lines in the first row). For hybrid coordinate cases, the cross section is taken along the first grid level, $k=1$, which intersects the near-surface inversion (fourth row of Figs. 3 and 4; dotted line denoting cross section location in third row is not visible).
Due to grid skewness, errors are concentrated in regions of vertical variation in the potential temperature (see Fig. 1); recall that vertical diffusion is turned off in these cases. When WRF's traditional terrain-following coordinate is used, errors occur around both the near-surface inversion and the tropopause (top two rows of Figs. 3 and 4). However when the hybrid coordinate is used, errors at the tropopause are mostly eliminated (bottom two rows of Figs. 3 and 4). Although errors 

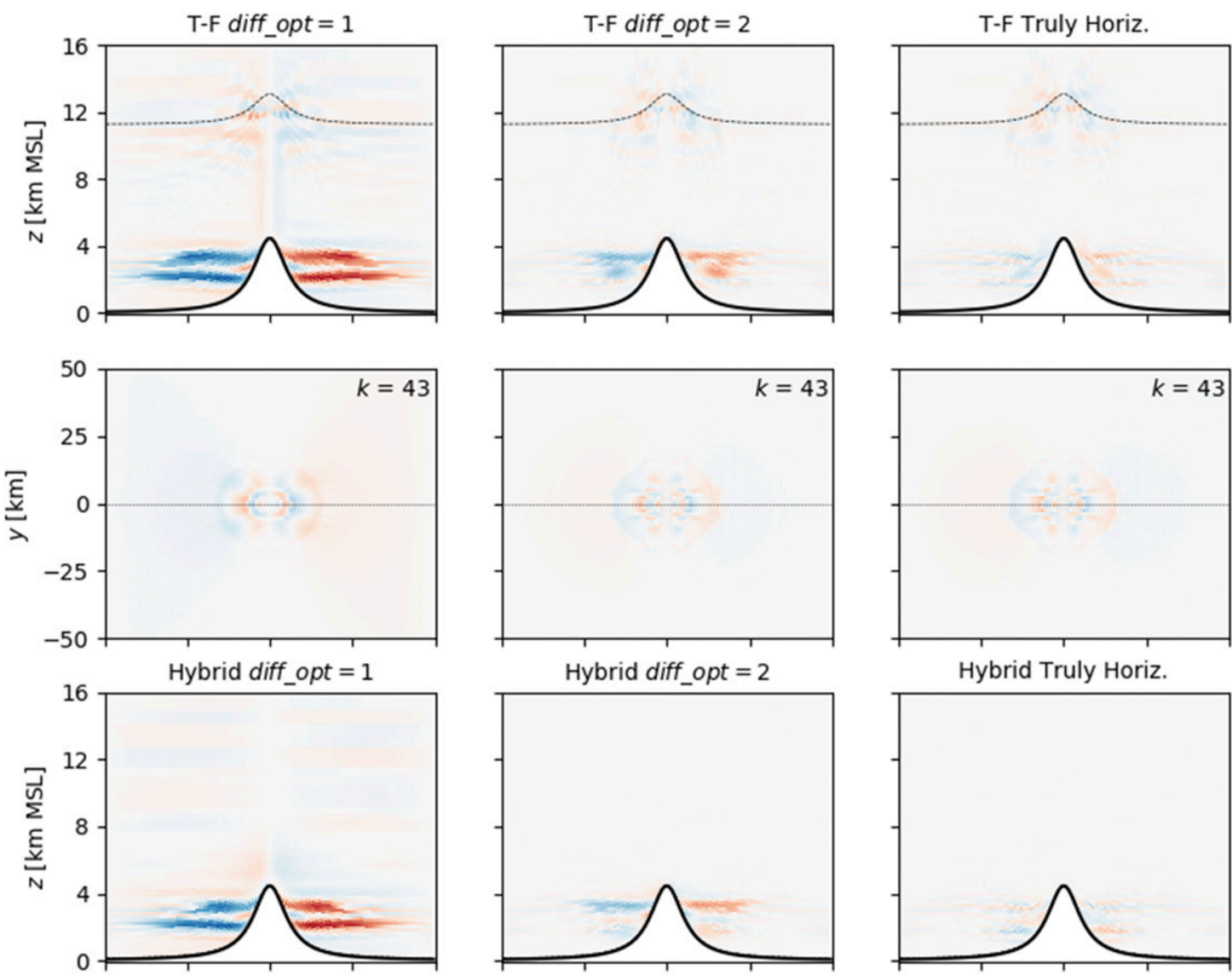

Hybrid diff_opt $=2$
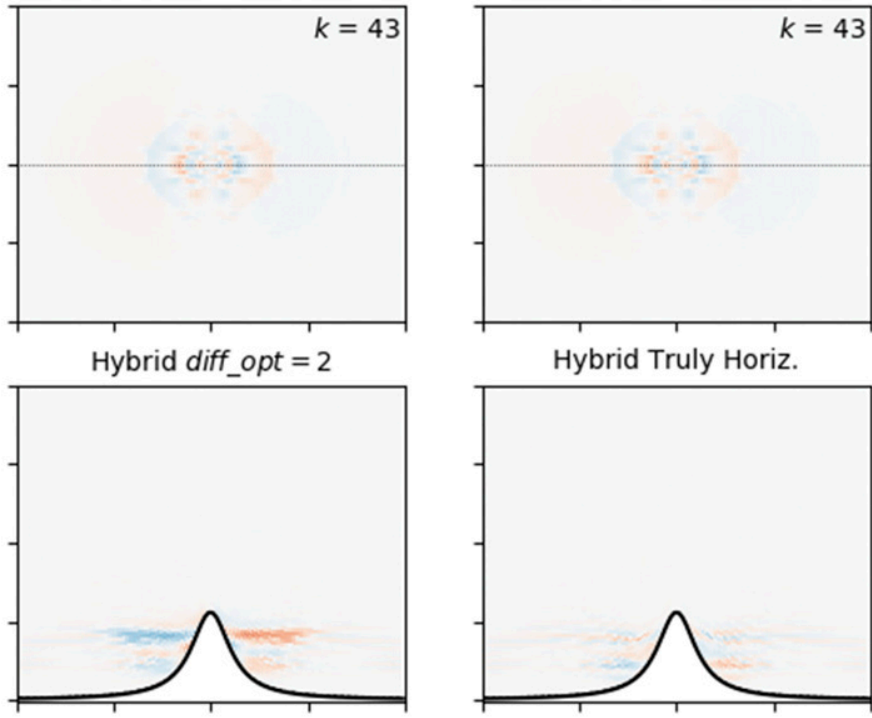

Hybrid Truly Horiz.
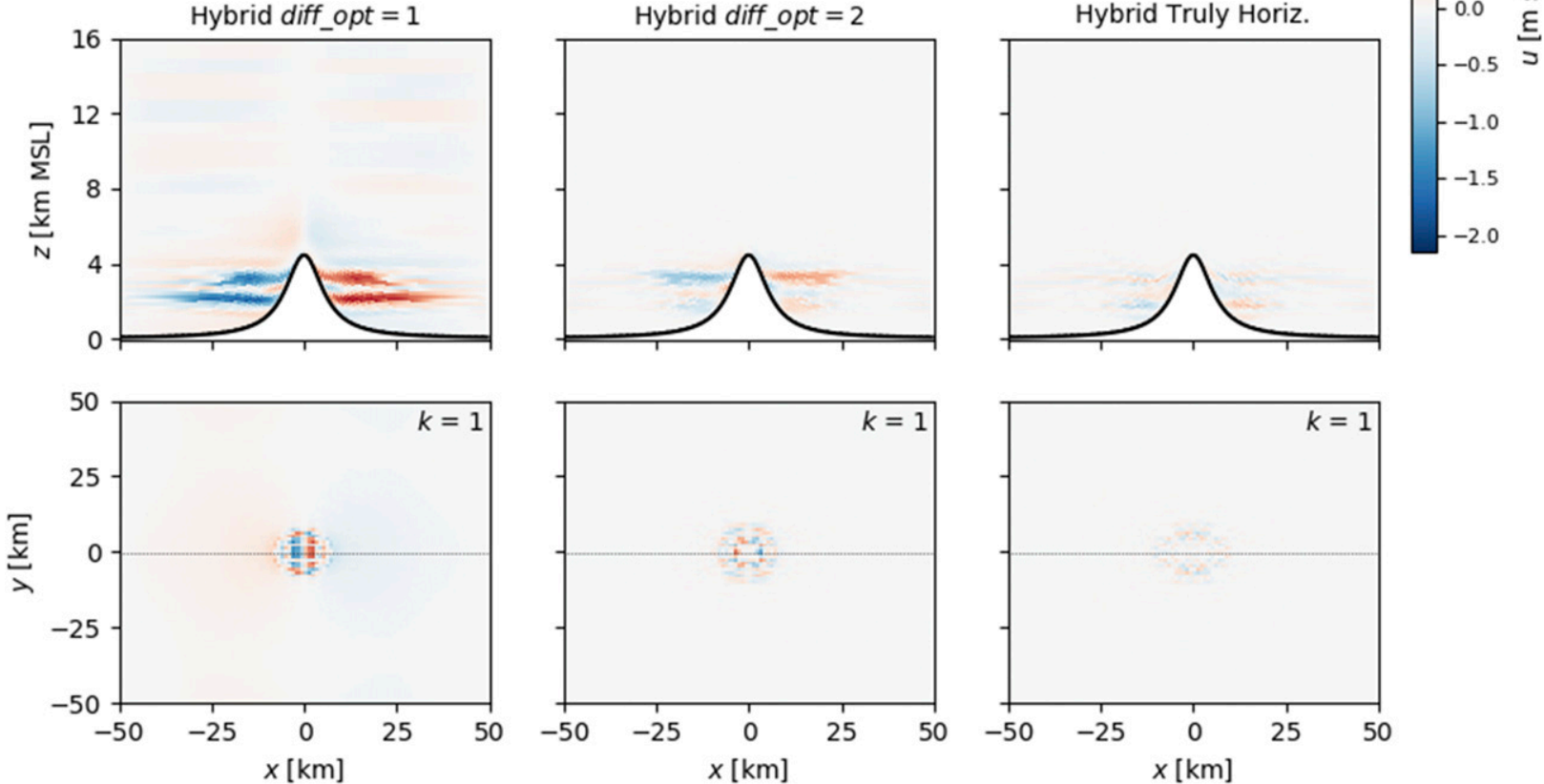

FIG. 4. Horizontal velocity field $u$ after $24 \mathrm{~h}$ of simulation time for standard atmosphere-at-rest test cases with large diffusivity. Results are shown for (left) diff_opt $=1$, (center) diff_opt $=2$, and (right) the present truly horizontal implementation; for (top two rows) WRF's traditional terrain-following coordinate and (bottom two rows) the hybrid coordinate. For each case, an $x-z$ cross section is shown through the $y$ centerline of the domain (first and third rows), and an $x-y$ cross section is shown along a specific vertical level (second and fourth rows). Dotted lines depict cross-section locations in corresponding plots. For terrain-following cases, the $x-y$ cross section is shown at $k=43$ (denoted by the dotted line in the first row), while for hybrid cases, it is shown at the first vertical level, $k=1$ (dotted line in third row not visible).

are still present when the truly horizontal potential temperature diffusion method is used, they generally follow the pattern of those for diff_opt $=2$, but with smaller magnitudes. Performance is especially good near the surface, where extrapolation is used to better predict the gradient when a truly horizontal grid point is not available. These results provide confidence in the present implementation.
Validation results are summarized in Table 1 for both the large and small diffusion options, where it can be seen that in general, progressing from diff_opt $=1$ to diff_opt $=2$ to the truly horizontal scheme reduces model error, often substantially.

For the large diffusivity cases, the maximum error in the potential temperature field over the entire domain after $24 \mathrm{~h}$ of 

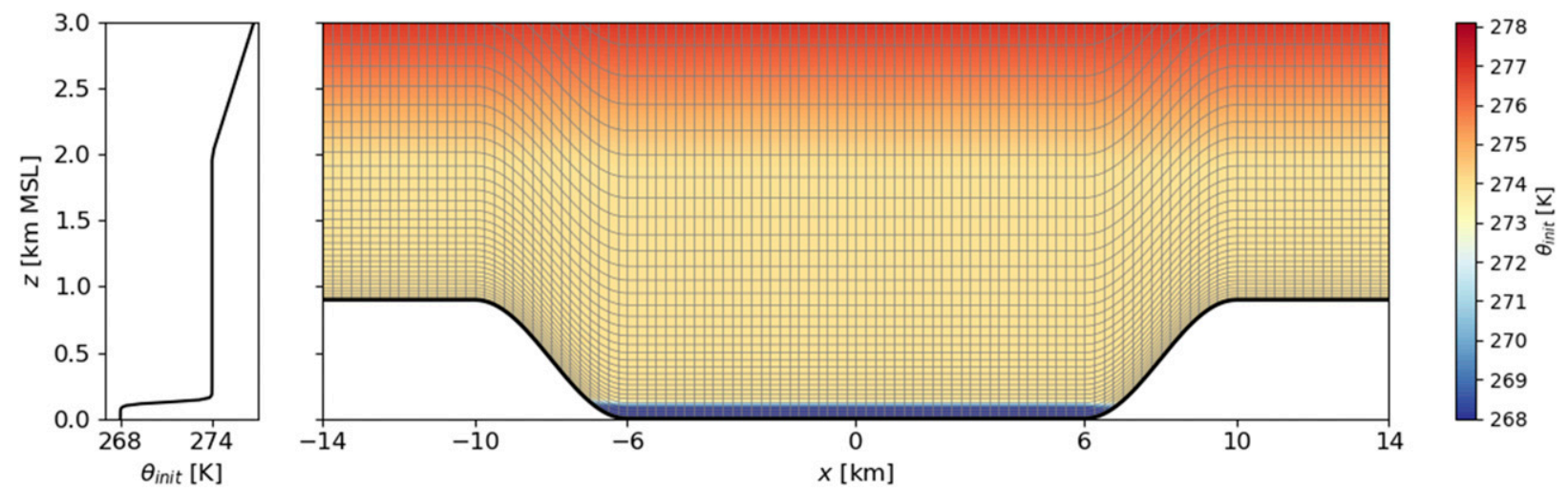

FIG. 5. (left) Initial potential temperature field $\theta_{\text {init }}$ and (right) grid setup for idealized cold-air pool cases, with every other grid line shown for clarity. Note that a limited vertical extent is shown to highlight the near-surface inversion; the full domain extends up to $z=20 \mathrm{~km}$ MSL.

simulation time is reduced by roughly a factor of 8 when switching from diff_opt $=1$ to diff_opt $=2$, with a more modest reduction between diff_opt $=2$ and the truly horizontal scheme. Model improvement between diffusion options is more evident when examining the velocity field. For the large diffusivity cases, the maximum horizontal velocity $|u|$ values are reduced by roughly $25 \%$ from diff_opt $=1$ to diff_opt $=2$, and nearly another $50 \%$ from diff_opt $=2$ to the truly horizontal scheme. Intuitively, the overall model errors are smaller in magnitude for the small diffusivity cases, although similar trends to the large diffusivity cases are found.

Note that the $u$ and $v$ velocity fields in each case are nearly identical, thus validating the full three-dimensional implementation; however, only $u$ results are shown here for brevity. Interestingly, while the use of diff_opt $=2$ improves upon diff_opt $=1$ in terms of the maximum horizontal velocity, vertical velocities $|w|$ actually increase for the large diffusivity case. This is due to large errors in $w$ along the steep terrain surface, and is related to WRF's kinematic boundary condition, which requires extrapolation of the horizontal velocities $u$ and $v$ to the surface. Nonetheless, it highlights the limits of the standard diff_opt $=2$ implementation, in which the metric terms $\left[z_{x}\right.$ in Eqs. (4) and (5)] are calculated using local grid cell values only. It should also be noted that, in each of the present atmosphere-at-rest cases, errors in the potential temperature field grow steadily with time, while errors in the velocity field tend to stabilize after several hours. Thus, while the relative performance of the methods is established after several hours of simulation time, the results reported here are specific to the chosen 24-h simulation time.

\section{b. Idealized cold-air pool case}

\section{1) Model SeTuP}

The truly horizontal potential temperature diffusion scheme is expected to improve WRF's ability to simulate cold-air pools by reducing spurious numerical mixing of the potential temperature field. To demonstrate this, the atmosphere-at-rest configuration is applied to an idealized CAP simulation. The CAP is initialized within a two-dimensional valley and allowed to evolve in the absence of any atmospheric or surface forcing.
Analytically, the CAP should persist, unchanged from the initial condition. However, numerical mixing leads to erosion of the CAP, as shown below for each horizontal diffusion option in WRF (diff_opt $=1$, diff_opt $=2$, and truly horizontal).

The idealized CAP setup follows that of Sheridan (2019). The valley topography is given by

$h(x)=\left\{\begin{array}{cc}0, & |x|<L_{b} / 2, \\ h_{v}\left\{1-\cos ^{2}\left[\frac{\pi\left(|x|-L_{b} / 2\right)}{L_{s}}\right]\right\}, & L_{b} / 2 \leq|x| \leq L_{b} / 2+L_{s}, \\ h_{v}, & |x|>L_{b} / 2+L_{s},\end{array}\right.$

where $h_{v}=900 \mathrm{~m}$ is the valley depth, $L_{b}=12 \mathrm{~km}$ is the width of the flat valley bottom, and $L_{s}=4 \mathrm{~km}$ is the width of the slope on either side of the valley (see Fig. 5).

The domain is $28 \mathrm{~km}$ wide with $\Delta x=125 \mathrm{~m}$ and periodic lateral boundary conditions. The domain height is $20 \mathrm{~km}$. The vertical grid spacing $\Delta z$ is $10 \mathrm{~m}$ at the first grid level above the surface; it is then stretched by a factor $r=1.04$ such that $\Delta z \approx$ $130 \mathrm{~m}$ at $z \approx 3 \mathrm{~km}$, above which it is held constant. WRF's traditional terrain-following vertical coordinate is used. This setup is comparable to the "BSN" cases of Sheridan (2019) with $900 \mathrm{~m}$ valley depth. However, $L_{s}$ is reduced here by a factor of 2 such that the valley sidewalls are steep enough to test the truly horizontal diffusion scheme (the maximum slope is roughly $20^{\circ}$ ).

Although Sheridan (2019) generated the initial condition for CAP simulations by spinning up a one-dimensional atmospheric model, a simplified initial condition is used here. The potential temperature profile is given by

$\theta_{\text {init }}(z)=\left\{\begin{array}{cc}\theta_{0}+\frac{\Delta \theta}{2}\left\{\tanh \left[\frac{2\left(z-z_{\text {inv }}\right)}{d_{\text {inv }}}\right] \tanh ^{-1}(0.99)-1\right\}, & z<z_{r}, \\ \theta_{0}\left[\frac{N^{2}}{g\left(z-z_{r}\right)}+1\right], & z \geq z_{r},\end{array}\right.$

where $\theta_{0}=274 \mathrm{~K}$ is the background atmospheric temperature, $\Delta \theta=6 \mathrm{~K}$ is the change in temperature in the CAP, $z_{\text {inv }}=125 \mathrm{~m}$ 

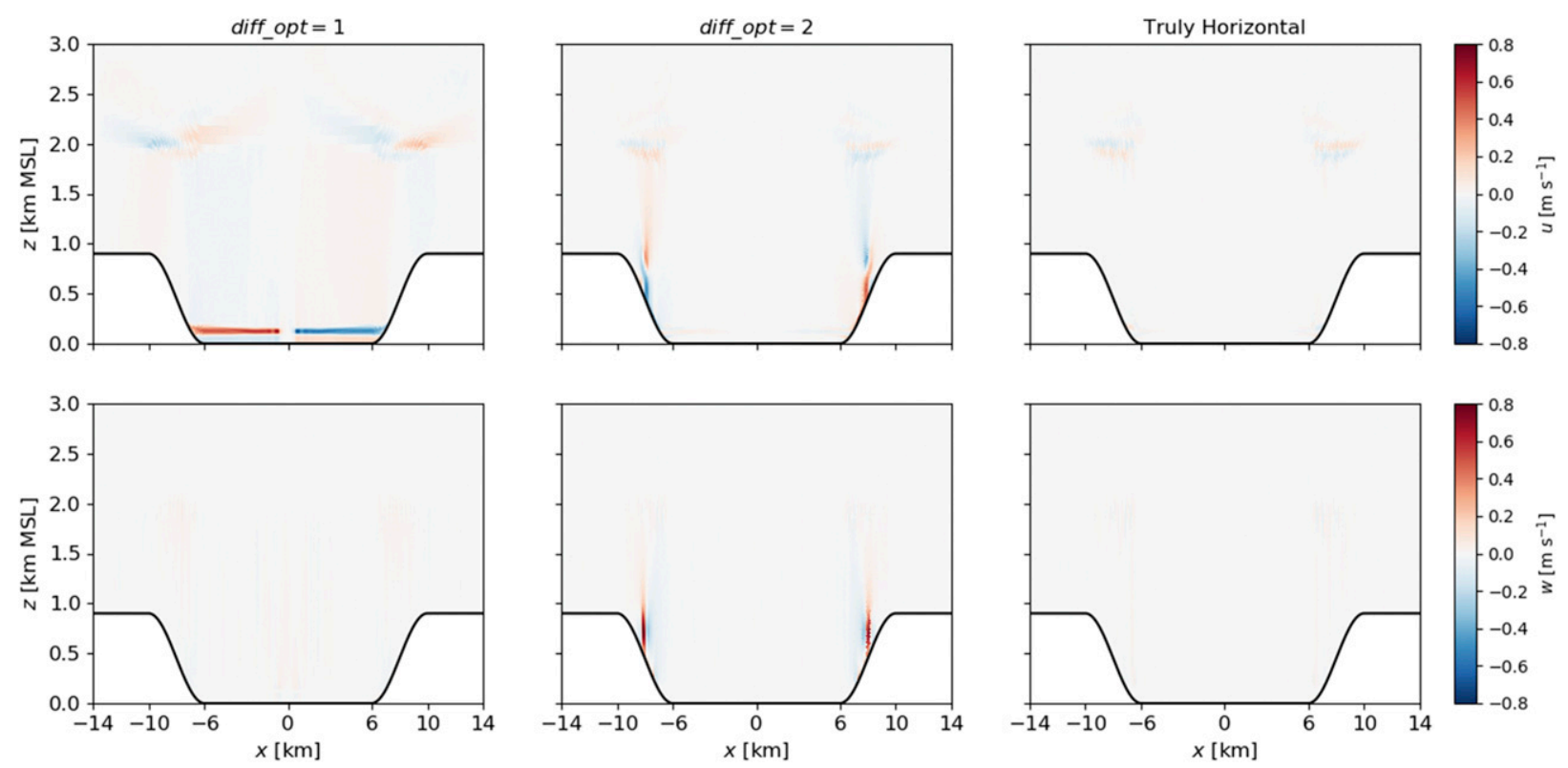

FIG. 6. (top) Horizontal $(u)$ and (bottom) vertical $(w)$ velocity fields after $1.5 \mathrm{~h}$ of simulation time for idealized cold-air pool cases. Results are shown for (left) diff_opt $=1$, (center) diff_opt $=2$, and (right) the truly horizontal scheme.

is the height of the CAP inversion, $d_{\text {inv }}=100 \mathrm{~m}$ is the vertical scale of the CAP inversion, $z_{r}=2 \mathrm{~km}$ is the height of the residual (neutral) layer top, and $N=0.01 \mathrm{~s}^{-1}$ is the BruntVäisälä frequency of the ambient stable layer aloft. This is a close approximation of the initial profile (after spinup) used by Sheridan (2019) for "N001" cases, see Fig. 3a therein.

As in the standard atmosphere-at-rest case, the initial velocity is zero, the atmosphere is dry, and all atmospheric physics modules are turned off. The horizontal diffusion coefficient for heat $K_{H}^{h}$ is set to a constant value of $10 \mathrm{~m}^{2} \mathrm{~s}^{-1}$, while all other diffusion coefficients are set to zero. This setup is representative of quiescent CAP conditions with minimal wind-driven mixing, and is meant to highlight the performance of the horizontal potential temperature diffusion scheme. A fully realistic CAP scenario that includes the effect of winds on CAP dynamics is presented below in section 4 .

\section{2) Results}

Similar to the standard atmosphere-at-rest case, numerical errors cause spurious flows to develop in the idealized CAP case as well (Fig. 6). The errors are present both in the valley basin, where the CAP inversion is present, and aloft, because grid skewness due to the valley topography extends high into the domain (see grid lines in Fig. 5). It is possible that the artificial flow structures depicted in Fig. 6 may be hidden in a more realistic CAP simulation with other forcing mechanisms. However, these simulations highlight potential sources of error in modeling CAP dynamics in WRF, or other models with similar treatment of horizontal diffusion.

When diff_opt $=1$ is used, coherent spurious horizontal flow develops along the CAP inversion, with a maximum velocity of roughly $0.8 \mathrm{~m} \mathrm{~s}^{-1}$. The simulations are stopped when this flow reaches the middle of the domain, after $1.5 \mathrm{~h}$. Weak spurious vertical circulations also develop within the residual layer, up to roughly $2 \mathrm{~km}$ (Fig. 6, left column). While the use of diff_opt $=2$ reduces spurious flow along the CAP inversion, a stronger spurious circulation, again with a maximum of roughly $0.8 \mathrm{~m} \mathrm{~s}^{-1}$, develops on the valley sidewalls and extends vertically up through the residual layer (Fig. 6, middle column). For both standard diffusion options, nonphysical transport of heat along the valley sidewalls near the CAP inversion leads to spurious temperature variability and flow within the residual layer, although it was initially neutral. As seen previously in the basic atmosphere-at-rest case, the strong vertical flows that develop with diff_opt $=2$, but not with diff_opt $=1$, are caused by the limited implementation of WRF's metric terms $\left[z_{x}\right.$ in Eqs. (4) and (5)], which cannot fully account for grid skewness in this case. Application of the truly horizontal diffusion scheme minimizes the errors observed when using the standard WRF options, resulting in a mostly quiescent CAP (Fig. 6, right column).

Erosion of the CAP inversion is depicted in Fig. 7 for each diffusion option to demonstrate the potential effect of model errors on CAP persistence. The horizontally averaged potential temperature profile along the flat valley bottom, denoted $\bar{\theta}$, is compared to the initial profile, denoted $\theta_{\text {init }}$. Substantial numerical mixing occurs with diff_opt $=1$, with a maximum change of roughly $0.5 \mathrm{~K}$ on either side of the inversion. The use of diff_opt $=2$ reduces this mixing by roughly an order of magnitude. While $\bar{\theta}$ for the truly horizontal potential temperature diffusion case roughly overlaps that for diff_opt $=2$ in Fig. 7a, the change from $\theta_{\text {init }}$ is still reduced by a factor of 2-4 (Fig. 7b). A comparable improvement in numerical mixing, although smaller in overall magnitude, is seen at the top of the residual layer as well (not shown). Based on these results, the truly horizontal potential temperature diffusion scheme shows promise for improving CAP simulation, which is a long-standing 
(a)

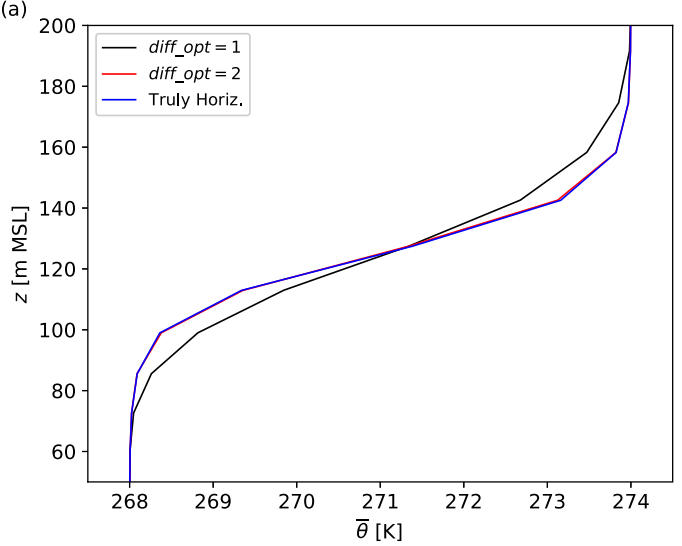

(b)

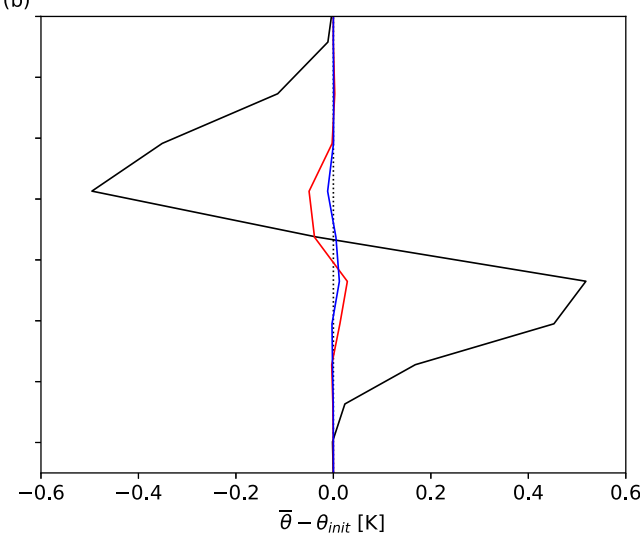

FIG. 7. Erosion of the near-surface inversion due to numerical mixing after $1.5 \mathrm{~h}$ of simulation time for idealized cold-air pool cases. Shown are (a) the horizontally averaged temperature along the flat valley bottom, $\bar{\theta}$, as well as (b) the difference between $\bar{\theta}$ and the initial profile $\theta_{\text {init }}$. For clarity in (a), $\theta_{\text {init }}$ is not shown because it essentially overlaps the blue $\bar{\theta}$ curve for the truly horizontal case.

model forecast challenge (Mahrt 1998). This technique is applied to a realistic CAP case in the next section.

\section{Realistic cold-air pool simulation}

\section{a. Model setup}

To test the truly horizontal potential temperature diffusion scheme in a realistic WRF setup, a cold-start reforecast is completed for 0000-2400 UTC 12 January 2017 corresponding to 1600 Pacific standard time (PST) 11 January-1600 PST 12 January (PST $=$ UTC $-8 \mathrm{~h}$ ). This day was chosen from the WFIP2 event log (see Wilczak et al. 2019), which documents meteorological events observed during the field campaign and is available through the DAP [Atmosphere to Electrons (A2e) 2017a], because it is in the middle of a multiday persistent CAP event, providing relatively steady conditions for testing. Although the CAP did experience some diurnal variation, it did not experience large variations in synoptic forcing, as occurred at the beginning and end of the multiday event. Furthermore, this day is representative of common cold pool conditions within the Columbia basin, as well as a common model bias in which wind speeds within the CAP are overestimated by the provisional WFIP2 HRRR model [see Atmosphere to Electrons (A2e) 2017a]. It is hypothesized that by using the truly horizontal diffusion scheme, numerical mixing of the temperature field will be reduced, leading to a reduction in this positive wind speed bias within the CAP.

The CAP case is run in WRF version 4.1.2. The WFIP2 provisional HRRR/HRRRNEST domains are used, with horizontal grid spacings of $\Delta x=3 \mathrm{~km}$ on the outer domain, $\mathrm{d} 01$ (as in the standard HRRR model), and $\Delta x=750 \mathrm{~m}$ on the inner domain, d02, as shown in Fig. 8. The nested domain, $\mathrm{d} 02$, is initialized $3 \mathrm{~h}$ after $\mathrm{d} 01$, and one-way nesting is employed such that the results on $\mathrm{d} 02$ do not feed back to d01. The WFIP2 "control” configuration, which corresponds

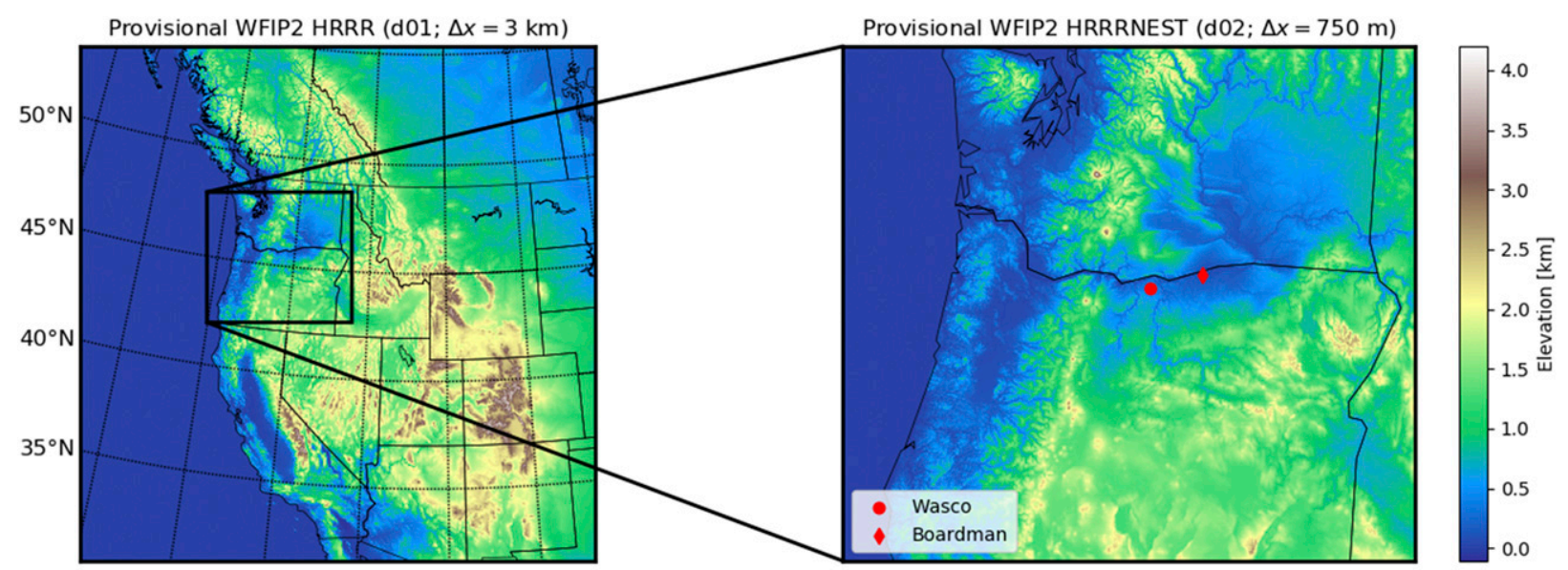

$125^{\circ} \mathrm{W} \quad 120^{\circ} \mathrm{W} 115^{\circ} \mathrm{W} 110^{\circ} \mathrm{W} 105^{\circ} \mathrm{W}$

FIG. 8. Domain setup for real cold-air pool cases, including the locations of the Wasco and Boardman observation sites. The provisional WFIP2 (left) HRRR and (right) HRRRNEST domains are used in a one-way nesting setup following Olson et al. (2019). 


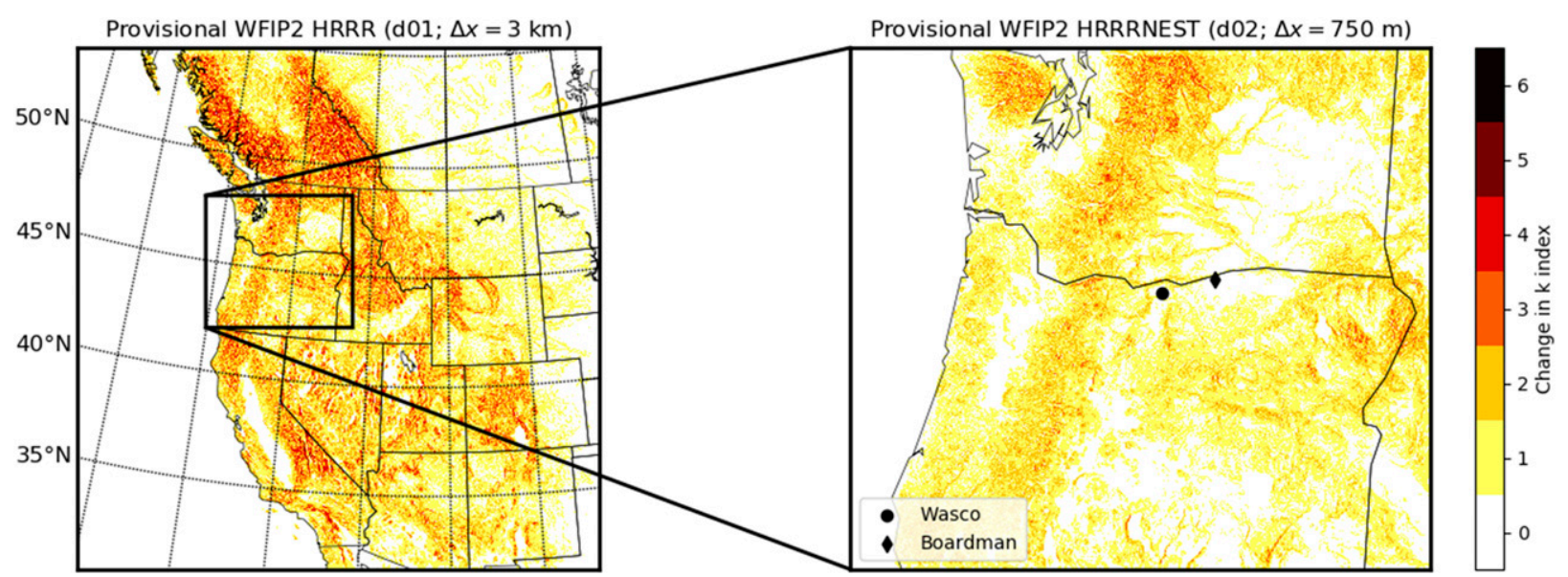

$125^{\circ} \mathrm{W} \quad 120^{\circ} \mathrm{W} \quad 115^{\circ} \mathrm{W} \quad 110^{\circ} \mathrm{W} \quad 105^{\circ} \mathrm{W}$

FIG. 9. Maximum change in $k$ index necessary for a truly horizontal gradient calculation at each horizontal grid point on the provisional WFIP2 (left) HRRR and (right) HRRRNEST domains. The locations of the Wasco and Boardman observation sites are shown for reference.

to the standard HRRR configuration, is used following Olson et al. (2019), see Table 2 therein. Notably, this configuration includes WRF's traditional terrain-following vertical coordinate (hybrid_opt $=0$ ). The HRRR physics suite is employed, including the Mellor-Yamada-Nakanishi-Niino (MYNN; Nakanishi and Niino 2006) Level 2.5 planetary boundary layer (PBL) scheme (bl_pbl_physics = 5) and associated surface layer scheme (sf_sfclay_physics $=5$ ), horizontal Smagorinsky closure $\left(\mathrm{km} \_\right.$opt $\left.=4\right)$, the Rapid Update Cycle (RUC) land surface model (sf_surface_physics $=3$ ), the Thompson aerosol-aware microphysics scheme (mp_ physics = 28; Thompson and Eidhammer 2014), and the RRTMG radiation schemes (ra_sw/lw_physics $=4$; Iacono et al. 2008). The WRF input and boundary files for this setup are available through the DAP [Atmosphere to Electrons (A2e) 2017f].

Three model runs are completed, varying only the horizontal diffusion scheme: option 1 uses (diff_opt $=1$ ), as in the standard WFIP2 control configuration, option 2 uses (diff_opt $=2$ ), as in the WFIP2 "experimental" configuration (Olson et al. 2019), and option 3 uses the newly implemented truly horizontal diffusion scheme for potential temperature. The same horizontal diffusion scheme is used on each model domain. Note that for option 3, horizontal diffusion of prognostic variables other than the potential temperature is calculated as with diff_opt $=2$.

In each case, WRF's option for adding positive-definite sixth-order horizontal diffusion (diff_6th_opt $=2$ ) is used with a factor of 0.25 , also on both domains. However, sixthorder diffusion is reduced over sloping terrain using diff_ 6th_slopeopt $=1$ with a threshold value of diff_6th_thresh $=$ 0.05 . Thus, the sixth-order filter is linearly damped between slopes of 0 and $0.05\left(2.86^{\circ}\right)$, and shut off for larger slopes. This feature was introduced in WRF as of version 4 to prevent overdiffusion in regions of sloping terrain, where errors related to the horizontal diffusion scheme are expected to be relatively large.
Two specific features of the WFIP2 provisional HRRR domains contribute to errors associated with the horizontal diffusion scheme. For one, both domains resolve steep terrain slopes, with maxima of roughly $20^{\circ}$ on $\mathrm{d} 01$ and $33^{\circ}$ on d02. Note that due to the use of single pass smoothing on the terrain of the provisional WFIP2 HRRR model, the maximum slope on $\mathrm{d} 01$ is slightly lower than that presented above for the standard HRRR model of $26^{\circ}$. Additionally, both domains have large near-surface grid aspect ratios $\alpha=$ $\Delta x / \Delta z$ due to relatively fine near-surface vertical resolution ( $\Delta z \approx 16 \mathrm{~m}$ for the first grid level), which is necessary for surface layer parameterizations.

The potential for error on both domains is illustrated in Fig. 9, which shows, for each horizontal grid point, the maximum change in vertical index $k$ necessary for a truly horizontal gradient calculation. As discussed previously, a change in $k$ of 1 or greater will lead to errors if the standard diff_opt $=2$ scheme is used (Figs. 1b,c). The maximum change in $k$ is 6 on d01 and 4 on d02, with values of 1 or greater prevalent on both domains. Thus, the truly horizontal scheme implemented here is expected to reduce errors in the provisional WFIP2 HRRR setup.

\section{b. Comparison to observations}

Model results are compared to observations from the WFIP2 field campaign, focusing on two sites within the Columbia River basin (Wasco and Boardman, Oregon; see Fig. 8). Each site included a $915 \mathrm{MHz}$ radar wind profiler (RWP) that gathered hourly average horizontal wind speed and direction data with roughly $100 \mathrm{~m}$ vertical resolution up to several kilometers AGL. The lowest available data point in the RWP dataset is roughly $100 \mathrm{~m}$ AGL, thus data from collocated sodars and surface stations are used to fill in near-surface wind observations (see Table 2). Surface and sodar data are hourly averaged to match the frequency of the RWP data. Each dataset is available through the DAP, as detailed in Table 2. The "reviewed" datasets used here have been quality 
TABLE 2. Observation datasets from the WFIP2 field campaign used for model comparison. These datasets have been quality controlled (denoted "b0" in the DAP code), although the raw data are also available. Note that sodar data are generally available every $10 \mathrm{~m}$ between 30 and $200 \mathrm{~m}$ AGL; however, only data below the range of the RWP are used here.

\begin{tabular}{|c|c|c|c|c|c|c|}
\hline \multirow[b]{2}{*}{ Instrument } & \multicolumn{3}{|c|}{ Wasco } & \multicolumn{3}{|c|}{ Boardman } \\
\hline & $\begin{array}{l}\text { Vertical range } \\
\text { (m AGL) }\end{array}$ & DAP code & Reference & $\begin{array}{l}\text { Vertical range } \\
\text { (m AGL) }\end{array}$ & DAP code & Reference \\
\hline Surface station & 10 & met.z06.b0 & $\begin{array}{l}\text { Atmosphere to Electrons } \\
\text { (A2e) (2017b) }\end{array}$ & 10 & met.z12.b0 & $\begin{array}{l}\text { Atmosphere to Electrons } \\
\text { (A2e) }(2017 \mathrm{c})\end{array}$ \\
\hline Sodar & $30-70$ & sodar.z09.b0 & $\begin{array}{l}\text { Atmosphere to Electrons } \\
\text { (A2e) }(2017 \mathrm{~g})\end{array}$ & $30-110$ & sodar.z16.b0 & $\begin{array}{l}\text { Atmosphere to Electrons } \\
\text { (A2e) }(2017 \mathrm{~h})\end{array}$ \\
\hline $\begin{array}{l}\text { Radar wind } \\
\text { profiler (RWP) }\end{array}$ & $81-2542$ & radar.z04.b0 & $\begin{array}{l}\text { Atmosphere to Electrons } \\
\text { (A2e) }(2017 \mathrm{~d})\end{array}$ & $124-2156$ & radar.z07.b0 & $\begin{array}{l}\text { Atmosphere to Electrons } \\
\text { (A2e) }(2017 \mathrm{e})\end{array}$ \\
\hline
\end{tabular}

controlled, as detailed in the DAP, although the raw data are also available.

The CAP, which persists throughout the case study period, is characterized by predominantly easterly-northeasterly flow within the basin and southerly-southwesterly flow aloft (see Figs. 10a,b). This flow structure is common for CAP events in the Columbia basin [see Atmosphere to Electrons (A2e) 2017a]. Because radar-based temperature measurements were unreliable in cloudy cold pool conditions, the depth of the cold pool is best discerned from the change in wind direction, which occurs between 1 and $1.5 \mathrm{~km}$ AGL. The top of the cold pool is clearly visible at Wasco (Fig. 10a), but less so at Boardman (Fig. 10b) due to quality control issues. The results here focus on the wind speed within the cold pool, which is well captured in the observations.

The model bias $B$ is calculated for the horizontal wind speed $V$ as

$$
B_{V}=V_{\mathrm{WRF}}-V_{\mathrm{OBS}},
$$

such that a positive bias represents an overestimate of $V$ by the model, and a negative bias represents an underestimate. To compute the bias, WRF results are hourly averaged (with output every $15 \mathrm{~min}$ ) and linearly interpolated to the vertical levels of the observations. The bias is calculated similarly for the wind direction $\phi$. However, $360^{\circ}$ is subtracted from $B_{\phi}$ values greater than $180^{\circ}$, and $360^{\circ}$ is added to $B_{\phi}$ values less than $-180^{\circ}$, such that $B_{\phi}$ spans the range from $-180^{\circ}$ to $180^{\circ}$.

Model bias results are presented in Figs. 10c-h and summarized in Table 3. Note that Fig. 10 shows bias results for $\mathrm{d} 02$, although those for $\mathrm{d} 01$ are qualitatively similar. To allow for $3 \mathrm{~h}$ of model spinup time, and so that $\mathrm{d} 01$ and $\mathrm{d} 02$ results can be compared over the same time window, bias analysis is limited to 1900 PST 11 January 2017-1600 PST 12 January 2017. WRF tends to overestimate the wind speed below roughly $0.5 \mathrm{~km}$ AGL within the cold pool (red regions in Figs. 10c-h), especially during the night when the wind speed is elevated above this level (Figs. 10a,b, between roughly 0000-1000 PST, $0.5-1.5 \mathrm{~km} \mathrm{AGL).} \mathrm{This} \mathrm{suggests}$ that numerical mixing of high momentum from aloft is playing a role in the model bias.

Applying the truly horizontal potential temperature diffusion scheme consistently reduces the maximum wind speed bias, as compared to WRF's two standard options. This is true at both observation sites and for both WRF domains, with the reduction ranging from $8 \%$ to $26 \%$ on $\mathrm{d} 01$ and from $2 \%$ to $6 \%$ on $\mathrm{d} 02$. Use of the truly horizontal scheme also generally reduces the mean positive wind speed bias, by a range of $5 \%-22 \%$ on $\mathrm{d} 01$, and $12 \%-17 \%$ on $\mathrm{d} 02$. Note that the mean positive wind speed bias on $\mathrm{d} 02$ at Boardman increases slightly between diff_opt $=2$ and the truly horizontal scheme, although both of these options still represent an improvement over diff_opt $=1$.

The truly horizontal potential temperature diffusion scheme does not have a large effect on the wind direction bias. Although the mean wind direction bias generally increases slightly from diff_opt $=1$, to diff_opt $=2$, to the truly horizontal scheme, wind direction errors are roughly comparable for all model runs, as seen in the wind vectors in Figs. 10c-h and in Table 3. Due to channeling of the flow by the complex terrain of the Columbia basin, wind direction errors are likely affected by the terrain resolution, and are therefore more difficult to model accurately at the scale of the WFIP2 HRRR/HRRRNEST.

A more detailed view of the wind speed at Wasco (Fig. 11) reveals several important aspects of model performance during the CAP event. Most notably, performance improvements related to the horizontal diffusion scheme are concentrated within the bottom $1 \mathrm{~km}$ AGL, as seen in the vertical wind speed profiles in Figs. 11d and 11e. Positive bias is generally reduced below $0.5 \mathrm{~km}$ AGL, as corroborated by the time series of wind speed at $z \approx 250 \mathrm{~m}$ AGL in Fig. 11b. To a lesser extent, negative wind speed bias between 0.5 and $1 \mathrm{~km}$ AGL is also reduced by improving the horizontal diffusion scheme (Figs. 11d,e). Note that as in Fig. 10, Fig. 11 shows results for d02, although those for d01 are qualitatively similar.

Above $1 \mathrm{~km}$ AGL, all three schemes perform similarly (Figs. 11d,e), with large negative bias, as seen in Fig. 10). The minimum wind speed bias is roughly equal for all three diffusion options, ranging from -7 to $-5 \mathrm{~m} \mathrm{~s}^{-1}$ depending on the site and domain. Large negative biases tend to occur above the cold pool, where the model grid is less skewed and there is less vertical temperature variability, and thus the error cannot be mitigated through the use of the truly horizontal potential temperature diffusion scheme. Modifications to the $\mathrm{PBL}$ 

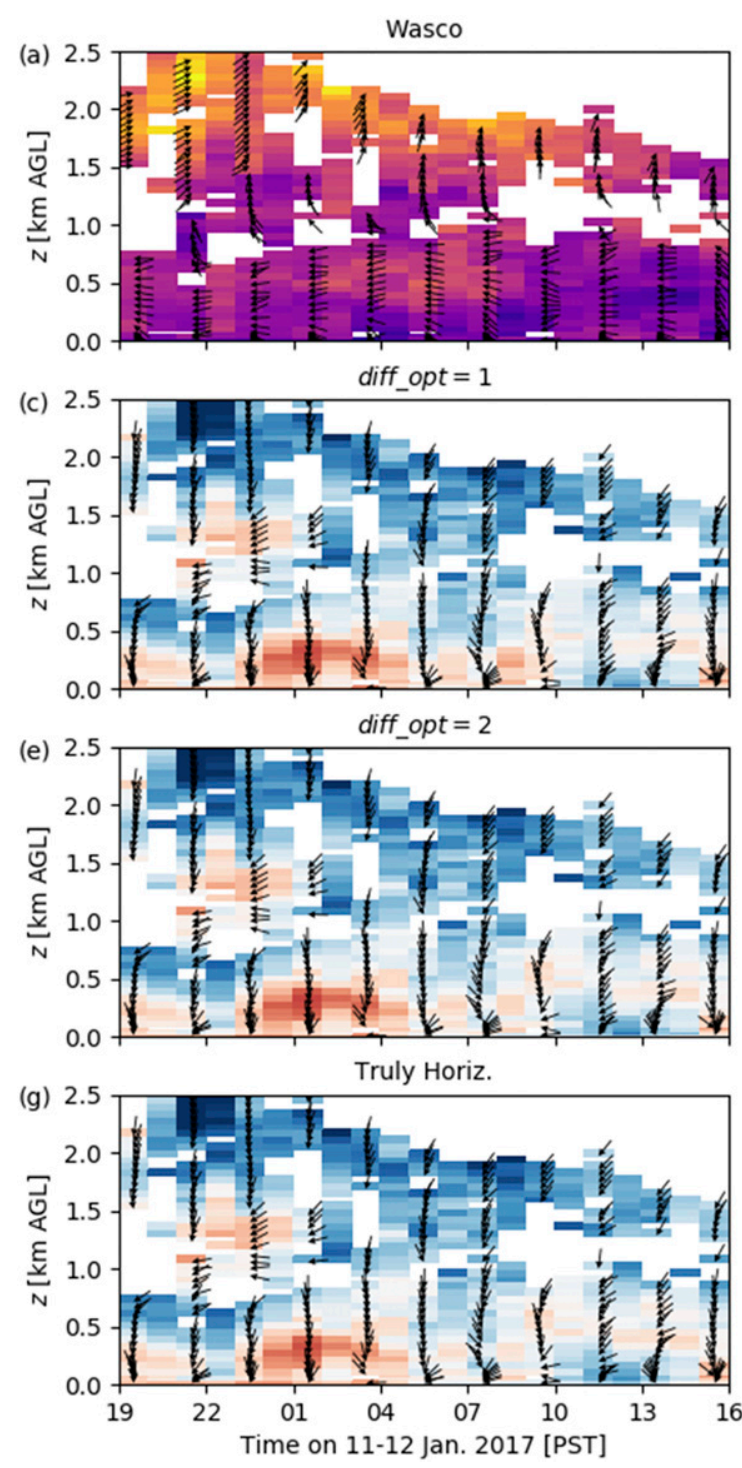

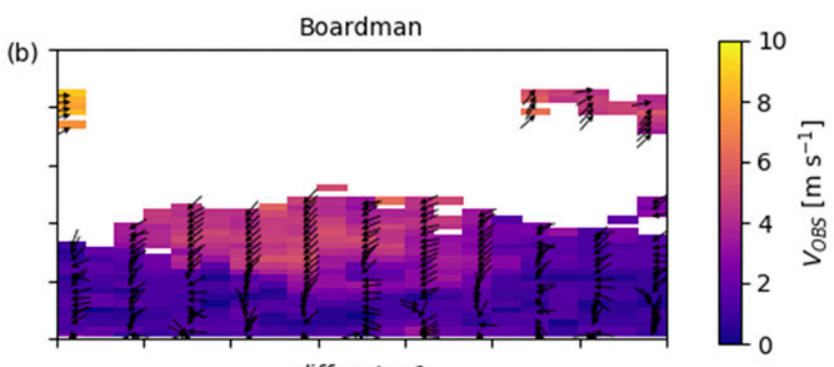

(d)
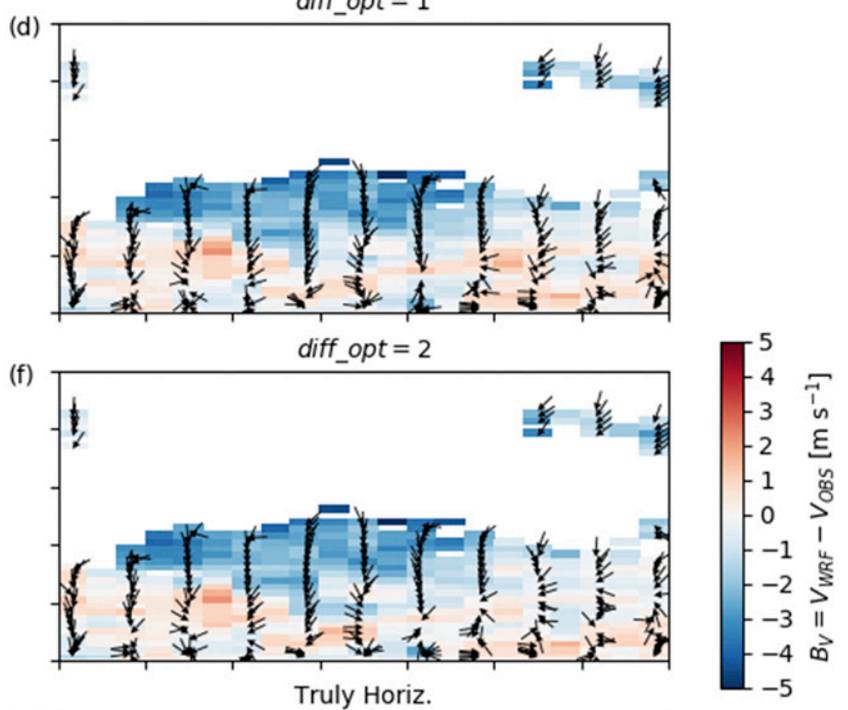

(h)

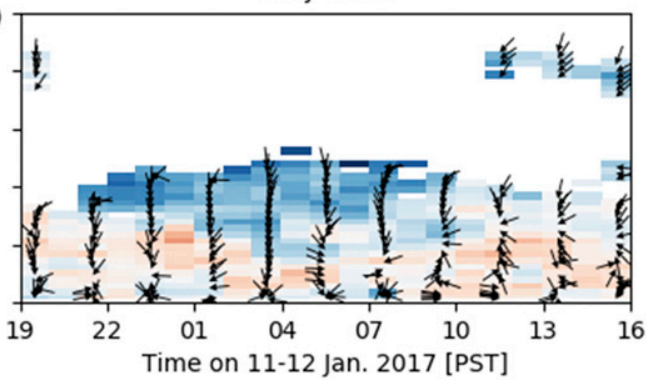

FIG. 10. Wind speed and wind direction bias results for real cold-air pool cases. Results are shown for the provisional WFIP2 HRRRNEST domain (d02) at the (left) Wasco and (right) Boardman observation sites. (a),(b) The observed wind speed $V_{\text {OBS }}$ and wind direction $\phi_{\mathrm{OBS}}$ at each site using the datasets shown in Table 2. (c)-(h) The wind speed bias $B_{V}$ and the wind direction bias $B_{\phi}$, as calculated in Eq. (12), for each horizonal diffusion option. $B_{\phi}$ spans the range from $-180^{\circ}$ to $180^{\circ}$, with $0^{\circ}$ depicted as northerly and $\pm 180^{\circ}$ depicted as southerly. Wind direction results are shown every other hour for clarity.

treatment or other model components are likely necessary to reduce negative wind speed bias aloft.

Very near to the surface, improving the diffusion scheme tends to reduce the modeled wind speed. However, this does not always reduce the model bias, as in Fig. 11c, which shows wind speed time series at $10 \mathrm{~m}$ AGL. This is expected to a certain extent, because unlike in the idealized cases, the $10 \mathrm{~m}$ wind speed is highly dependent on factors other than the modeled temperature field. Moreover, mesoscale models like the provisional WFIP2 HRRR/HRRRNEST are inherently limited in their ability to resolve near-surface features, especially in stable conditions. For example, Fig. 11e depicts an observed near-surface jet of roughly $4 \mathrm{~m} \mathrm{~s}^{-1}$. Although diff_opt $=2$ and the truly horizontal scheme appear to capture the jet more accurately, it is only partially resolved in the model, regardless of the diffusion scheme. Near-surface model evaluation should be considered in the context of these finescale features.

\section{Summary and conclusions}

In this work, a truly horizontal diffusion scheme for potential temperature has been added to the Weather Research and Forecasting Model. The method, which follows Mahrer (1984), uses Taylor series approximations to vertically interpolate variables to the level at which a truly horizontal gradient can be 
TABLE 3. Summary of model bias [Eq. (12)] results for real cold-air pool cases, with $B_{V}$ values in $\mathrm{m} \mathrm{s}^{-1}$ and $B_{\phi}$ values in degrees (spanning $-180^{\circ}$ to $180^{\circ}$ ). The bias is calculated using the observation datasets shown in Table 2 , and model results are hourly averaged and linearly interpolated to the vertical levels of the observations.

\begin{tabular}{|c|c|c|c|c|c|c|c|}
\hline \multirow[b]{2}{*}{ Domain } & \multirow[b]{2}{*}{ Option } & \multicolumn{3}{|c|}{ Wasco } & \multicolumn{3}{|c|}{ Boardman } \\
\hline & & $\operatorname{Max} B_{V}$ & Mean $B_{V} \geq 0$ & Mean $B_{\phi}$ & $\operatorname{Max} B_{V}$ & Mean $B_{V} \geq 0$ & Mean $B_{\phi}$ \\
\hline \multirow[t]{3}{*}{$\mathrm{d} 01$} & diff_opt $=1$ & 4.39 & 1.51 & 17.5 & 3.06 & 0.63 & 3.87 \\
\hline & diff_opt $=2$ & 4.22 & 1.25 & 19.6 & 3.05 & 0.59 & 4.92 \\
\hline & Truly horizontal & 3.90 & 1.17 & 20.1 & 2.25 & 0.56 & 8.96 \\
\hline \multirow[t]{3}{*}{$\mathrm{d} 02$} & diff_opt $=1$ & 3.28 & 1.03 & 19.5 & 2.23 & 0.69 & 9.23 \\
\hline & diff_opt $=2$ & 3.40 & 0.97 & 20.5 & 2.21 & 0.53 & 13.1 \\
\hline & Truly horizontal & 3.18 & 0.85 & 20.8 & 2.16 & 0.57 & 11.0 \\
\hline
\end{tabular}

calculated. This represents an improvement over WRF's standard horizontal diffusion options, in which horizontal gradients are calculated either along coordinate surfaces or in physical space using local metric terms.

Improvements in model performance were demonstrated by applying the newly implemented scheme to several test cases with steep and/or complex terrain. First, the scheme was validated in an idealized atmosphere-at-rest configuration, where the magnitude of numerically induced flows was reduced by roughly $50 \%$ or more compared to WRF's standard horizontal diffusion options. The atmosphere-at-rest configuration was then extended to an idealized cold-air pool case, where the truly horizontal scheme was shown to reduce errors that lead to spurious numerical mixing of the near-surface inversion.

After validation, the truly horizontal scheme was tested in a realistic WRF simulation of a cold-air pool in the Columbia River basin. Following Olson et al. (2019), a provisional setup of the operational HRRR model developed for the WFIP2 project was used. The setup includes two nested domains, the first with $3 \mathrm{~km}$ horizontal grid spacing, as in the HRRR, and the second with $750 \mathrm{~m}$ spacing. Through comparisons to wind profiles from two observation sites within the cold-air pool, the truly horizontal scheme was shown to reduce positive wind speed bias by up to roughly $20 \%$ relative to WRF's standard options. This reduction in model error should prove useful for wind energy forecasting during cold-air pool events, which was noted by Wilczak et al. (2019) as one of the greatest forecasting challenges in the complex terrain of the Columbia basin.

Based on the promising results of this study, future work on the truly horizontal diffusion scheme could be threefold. First, the method could be tested for additional complex-terrain weather scenarios, such as the gap flows and mountain waves that were consistently observed during the WFIP2 field campaign. Along these lines, the scheme could be included in retrospective forecast studies to more robustly test its ability to reduce model bias. Second, the method could be tested in conjunction with other WRF model improvements for complex terrain, such as new planetary boundary layer turbulence schemes (e.g., Kosović et al. 2020) or vertical grid nesting (Daniels et al. 2016). It could also be tested in models with similar vertical coordinate systems, such as the Model for Prediction Across Scales (MPAS; Skamarock et al. 2012).

Finally, the robustness of the method could be improved through the implementation of higher-order extrapolation schemes, especially near the surface, or through extension of the truly horizontal gradient calculation to other prognostic variables. Most directly, the truly horizontal diffusion scheme could be applied to moisture and other scalars. It could also be applied to velocity, including the calculation of eddy diffusion coefficients, although preliminary attempts (not presented here) showed little or no gain in model performance. Pressure gradient and advection calculations in WRF would likely also benefit from improved finite difference schemes; however, these implementations would be more complex than that presented here.

Additional methods for reducing errors related to grid skewness have been explored in the atmospheric modeling literature. Notable among these are immersed boundary methods (IBMs), which allow for the use of a non-terrainconforming mesh by applying surface boundary conditions along a terrain surface that intersects the model grid arbitrarily. There is potential for the truly horizontal scheme presented here to be used in combination with IBMs. For example, IBMs can represent finescale terrain features within a grid that follows the larger-scale terrain. Additionally, large pressure fluctuations can lead to grid deformation, even over flat terrain. However, in general, the use of an IBM should lessen the need for a truly horizontal gradient calculation. Moreover, while IBMs have shown promise in high-resolution large-eddy simulations (e.g., Ma and Liu 2017; DeLeon et al. 2018; Bao et al. 2018; Wiersema et al. 2020), they have only been tested at horizontal grid spacings up to roughly $100 \mathrm{~m}$ (Arthur et al. 2020). The extension of IBMs to mesoscale atmospheric models thus warrants further study.

Acknowledgments. Prepared by LLNL under Contract DE-AC52-07NA27344. This work was supported by the U.S. Department of Energy (DOE), Office of Energy Efficiency and Renewable Energy (EERE), Wind Energy Technologies Office (WETO). J.B.O. was also supported by the National Oceanic and Atmospheric Administration (NOAA)/Earth System Research Laboratories (ESRL) Atmospheric Science for Renewable Energy (ASRE) Program.

Data availability statement. All WRF input/boundary files and observation datasets used in section 4 are publicly available through the U.S. Department of Energy Data Archive and Portal (DAP; http://a2e.energy.gov/data), and are cited in the 

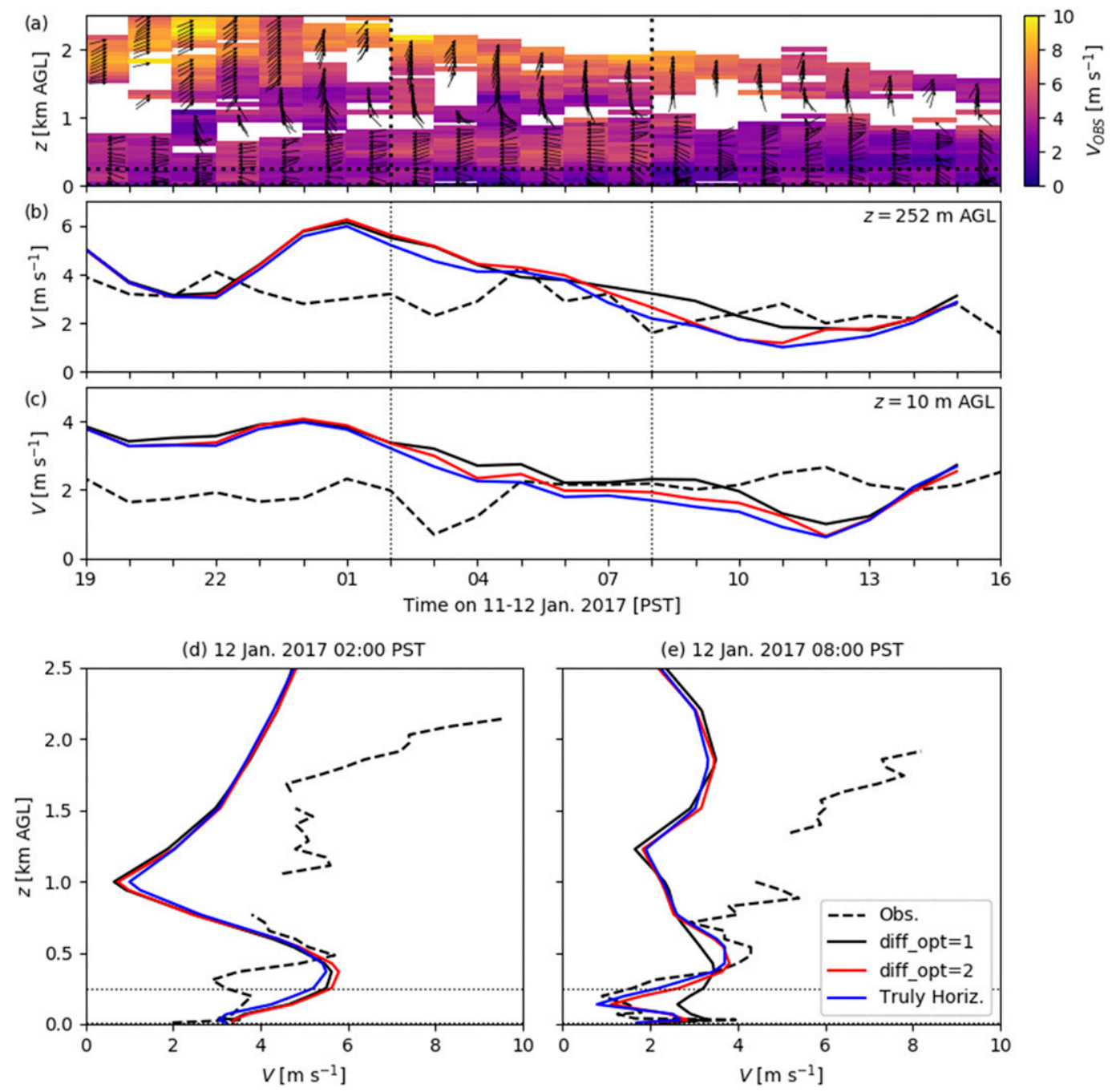

FIG. 11. Detailed view of modeled wind speed for real cold-air pool cases. Results are shown for the provisional WFIP2 HRRRNEST domain (d02) at the Wasco observation site. (a) The observed wind speed $V_{\text {OBS }}$ and wind direction $\phi_{\text {OBs }}$ using the datasets shown in Table 2. (b),(c) Time series of the observed and modeled wind speed $V$ at 252 and $10 \mathrm{~m}$ AGL, respectively. (d),(e) Profiles of the observed and modeled wind speed $V$ at 0200 and 0800 PST, respectively, 12 Jan 2017. Dotted vertical lines in (a)-(c) correspond to the times shown in (d) and (e), while dotted horizontal lines in (a), (d), and (e) correspond to the vertical levels shown in (b) and (c).

text. For additional information about the DAP, see Shaw et al. (2019).

\section{REFERENCES}

Arakawa, A., and V. R. Lamb, 1977: Computational design of the basic dynamical processes of the UCLA general circulation model. General Circulation Models of the Atmosphere, J. Chang, Ed., Vol. 17, Academic Press, 173-265.

Arthur, R. S., K. A. Lundquist, D. J. Wiersema, J. Bao, and F. K. Chow, 2020: Evaluating implementations of the immersed boundary method in the Weather Research and Forecasting Model. Mon. Wea. Rev., 148, 2087-2109, https://doi.org/ 10.1175/MWR-D-19-0219.1.

Atmosphere to Electrons (A2e), 2017a: wfip2/log.z01.00. A2e Data Archive and Portal, U.S. Department of Energy,
Office of Energy Efficiency and Renewable Energy, accessed 24 February 2020, https://doi.org/10.21947/1523403. , 2017b: wfip2/met.z06.b0. A2e Data Archive and Portal, U.S. Department of Energy, Office of Energy Efficiency and Renewable Energy, accessed 24 February 2020, https://doi.org/ $10.21947 / 1412526$.

—, 2017c: wfip2/met.z12.b0. A2e Data Archive and Portal, U.S. Department of Energy, Office of Energy Efficiency and Renewable Energy, accessed 24 February 2020, https://doi.org/ $10.21947 / 1412529$.

_ , 2017d: wfip2/radar.z04.b0. A2e Data Archive and Portal, U.S. Department of Energy, Office of Energy Efficiency and Renewable Energy, accessed 24 February 2020, https:// doi.org/10.21947/1412529.

— 2017e: wfip2/radar.z07.b0. A2e Data Archive and Portal, U.S. Department of Energy, Office of Energy Efficiency and 
Renewable Energy, accessed 24 February 2020, https:// doi.org/10.21947/1412529.

— , 2017f: wfip2/refcst.coldstart.icbc.02. A2e Data Archive and Portal, U.S. Department of Energy, Office of Energy Efficiency and Renewable Energy, accessed 24 February 2020, https://doi.org/10.21947/1405131.

— 2017g: wfip2/sodar.z09.b0. A2e Data Archive and Portal, U.S. Department of Energy, Office of Energy Efficiency and Renewable Energy, accessed 24 February 2020, https:// doi.org/10.21947/1412526.

— , 2017h: wfip2/sodar.z16.b0. A2e Data Archive and Portal, U.S. Department of Energy, Office of Energy Efficiency and Renewable Energy, accessed 24 February 2020, https:// doi.org/10.21947/1412529.

Bao, J., F. K. Chow, and K. A. Lundquist, 2018: Large-eddy simulation over complex terrain using an improved immersed boundary method in the Weather Research and Forecasting Model. Mon. Wea. Rev., 146, 2781-2797, https://doi.org/ 10.1175/MWR-D-18-0067.1.

Beck, J., and Coauthors, 2020: An evaluation of a hybrid, terrainfollowing vertical coordinate in the WRF-based RAP and HRRR models. Wea. Forecasting, 35, 1081-1096, https:// doi.org/10.1175/WAF-D-19-0146.1.

Benjamin, S. G., and Coauthors, 2016: A North American hourly assimilation and model forecast cycle: The Rapid Refresh. Mon. Wea. Rev., 144, 1669-1694, https://doi.org/10.1175/MWR-D-150242.1.

Billings, B. J., V. Grubišić, and R. D. Borys, 2006: Maintenance of a mountain valley cold pool: A numerical study. Mon. Wea. Rev., 134, 2266-2278, https://doi.org/10.1175/ MWR3180.1.

Daniels, M. H., K. A. Lundquist, J. D. Mirocha, D. J. Wiersema, and F. K. Chow, 2016: A new vertical grid nesting capability in the Weather Research and Forecasting (WRF) Model. Mon. Wea. Rev., 144, 3725-3747, https://doi.org/10.1175/ MWR-D-16-0049.1.

DeLeon, R., M. Sandusky, and I. Senocak, 2018: Simulations of turbulent flow over complex terrain using an immersedboundary method. Bound.-Layer Meteor., 167, 399-420, https://doi.org/10.1007/s10546-018-0336-8.

Dempsey, D., and C. Davis, 1998: Error analysis and tests of pressure gradient force schemes in a nonhydrostatic, mesoscale model. 12th Conf. on Numerical Weather Prediction, Phoenix, AZ, Amer. Meteor. Soc., 236-239.

Gal-Chen, T., and R. C. J. Somerville, 1975: On the use of a coordinate transformation for the solution of the Navier-Stokes equations. J. Comput. Phys., 17, 209-228, https://doi.org/ 10.1016/0021-9991(75)90037-6.

Grell, G. A., J. Dudhia, and D. R. Stauffer, 1994: A description of the fifth-generation Penn State/NCAR Mesoscale Model (MM5). NCAR Tech. Note NCAR/TN-398+STR, 121 pp., https://doi.org/10.5065/D60Z716B.

Iacono, M. J., J. S. Delamere, E. J. Mlawer, M. W. Shephard, S. A. Clough, and W. D. Collins, 2008: Radiative forcing by longlived greenhouse gases: Calculations with the AER radiative transfer models. J. Geophys. Res., 113, D13103, https://doi.org/ 10.1029/2008JD009944.

Janjić, Z. I., 1977: Pressure gradient force and advection scheme used for forecasting with steep and small scale topography. Beitr. Phys. Atmos., 50, 186-199.

, 1989: On the pressure gradient force error in $\sigma$-coordinate spectral models. Mon. Wea. Rev., 117, 2285-2292, https://oi.org/ 10.1175/1520-0493(1989)117<2285:OTPGFE>2.0.CO;2.
Klemp, J. B., 2011: A terrain-following coordinate with smoothed coordinate surfaces. Mon. Wea. Rev., 139, 2163-2169, https:// doi.org/10.1175/MWR-D-10-05046.1.

Kosović, B., P. J. Munoz, T. W. Juliano, A. Martilli, M. Eghdami, A. P. Barros, and S. E. Haupt, 2020: Three-dimensional planetary boundary layer parameterization for high-resolution mesoscale simulations. J. Phys. Conf. Series, 1452, 012080, https:// doi.org/10.1088/1742-6596/1452/1/012080.

Lareau, N. P., and J. D. Horel, 2015: Turbulent erosion of persistent cold-air pools: Numerical simulations. J. Atmos. Sci., 72, 1409-1427, https://doi.org/10.1175/JAS-D-14-0173.1.

—, E. Crosman, C. D. Whiteman, J. D. Horel, S. W. Hoch, W. O. J. Brown, and T. W. Horst, 2013: The persistent cold-air pool study. Bull. Amer. Meteor. Soc., 94, 51-63, https://doi.org/ 10.1175/BAMS-D-11-00255.1.

Lehner, M., and Coauthors, 2016: The METCRAX II field experiment: A study of downslope windstorm-type flows in Arizona's Meteor Crater. Bull. Amer. Meteor. Soc., 97, 217235, https://doi.org/10.1175/BAMS-D-14-00238.1.

Lu, W., and S. Zhong, 2014: A numerical study of a persistent cold air pool episode in the Salt Lake Valley, Utah. J. Geophys. Res. Atmos., 119, 1733-1752, https://doi.org/ 10.1002/2013JD020410.

Ma, Y., and H. Liu, 2017: Large-eddy simulations of atmospheric flows over complex terrain using the immersed-boundary method in the weather research and forecasting model. Bound.-Layer Meteor., 165, 421-445, https://doi.org/10.1007/s10546-017-0283-9.

Mahrer, Y., 1984: An improved numerical approximation of the horizontal gradients in a terrain-following coordinate system. Mon. Wea. Rev., 112, 918-922, https://doi.org/10.1175/15200493(1984)112<0918:AINAOT>2.0.CO;2.

Mahrt, L., 1998: Stratified atmospheric boundary layers and breakdown of models. Theor. Comput. Fluid Dyn., 11, 263279, https://doi.org/10.1007/s001620050093.

McCaffrey, K., and Coauthors, 2019: Identification and characterization of persistent cold pool events from temperature and wind profilers in the Columbia River basin. J. Appl. Meteor. Climatol., 58, 2533-2551, https://doi.org/10.1175/JAMC-D-19-0046.1.

Nakanishi, M., and H. Niino, 2006: An improved Mellor-Yamada level-3 model: Its numerical stability and application to a regional prediction of advection fog. Bound.-Layer Meteor., 119, 397-407, https://doi.org/10.1007/s10546-005-9030-8.

Olson, J. B., and Coauthors, 2019: Improving wind energy forecasting through numerical weather prediction model development. Bull. Amer. Meteor. Soc., 100, 2201-2220, https:// doi.org/10.1175/BAMS-D-18-0040.1.

Park, S.-H., W. C. Skamarock, J. B. Klemp, L. D. Fowler, and M. G. Duda, 2013: Evaluation of global atmospheric solvers using extensions of the Jablonowski and Williamson baroclinic wave test case. Mon. Wea. Rev., 141, 3116-3129, https://doi.org/ 10.1175/MWR-D-12-00096.1.

Pichugina, Y. L., and Coauthors, 2019: Spatial variability of winds and HRRR-NCEP model error statistics at three Dopplerlidar sites in the wind-energy generation region of the Columbia River basin. J. Appl. Meteor. Climatol., 58, 16331656, https://doi.org/10.1175/JAMC-D-18-0244.1.

Schär, C., O. Leuenberger, O. Fuhrer, D. Lüthi, and C. Girard, 2002: A new terrain-following vertical coordinate formulation for atmospheric prediction models. Mon. Wea. Rev., 130, 2459-2480, https://doi.org/10.1175/1520-0493(2002)130<2459: ANTFVC $>2.0 . \mathrm{CO} ; 2$.

Shaw, W. J., and Coauthors, 2019: The Second Wind Forecast Improvement Project (WFIP2): General overview. Bull. 
Amer. Meteor. Soc., 100, 1687-1699, https://doi.org/10.1175/ BAMS-D-18-0036.1.

Sheridan, P. F., 2019: Synoptic-flow interaction with valley cold-air pools and effects on cold-air pool persistence: Influence of valley size and atmospheric stability. Quart. J. Roy. Meteor. Soc., 145, 1636-1659, https://doi.org/10.1002/qj.3517.

Silcox, G. D., K. E. Kelly, E. T. Crosman, C. D. Whiteman, and B. L. Allen, 2012: Wintertime PM2. 5 concentrations during persistent, multi-day cold-air pools in a mountain valley. Atmos. Environ., 46, 17-24, https://doi.org/10.1016/j.atmosenv.2011.10.041.

Simmons, A. J., and D. M. Burridge, 1981: An energy and angularmomentum conserving vertical finite-difference scheme and hybrid vertical coordinates. Mon. Wea. Rev., 109, 758-766, https:// doi.org/10.1175/1520-0493(1981)109<0758:AEAAMC >2.0.CO;2.

Skamarock, W. C., and Coauthors, 2008: A description of the Advanced Research WRF version 3. NCAR Tech. Note NCAR/TN-475+STR, 113 pp., https://doi.org/10.5065/ D68S4MVH.

, J. B. Klemp, M. G. Duda, L. D. Fowler, S.-H. Park, and T. D. Ringler, 2012: A multiscale nonhydrostatic atmospheric model using centroidal Voronoi tesselations and C-grid staggering. Mon. Wea. Rev., 140, 3090-3105, https://doi.org/ 10.1175/MWR-D-11-00215.1.

— , and Coauthors, 2019: A description of the Advanced Research WRF model version 4. NCAR Tech. Note NCAR/ TN-556+STR, 145 pp., https://doi.org/10.5065/1dfh-6p97.

Thompson, G., and T. Eidhammer, 2014: A study of aerosol impacts on clouds and precipitation development in a large winter cyclone. J. Atmos. Sci., 71, 3636-3658, https://doi.org/ 10.1175/JAS-D-13-0305.1.

Vosper, S. B., and A. R. Brown, 2008: Numerical simulations of sheltering in valleys: The formation of nighttime cold-air pools. Bound.-Layer Meteor., 127, 429-448, https://doi.org/ 10.1007/s10546-008-9272-3.
Whiteman, C. D., and Coauthors, 2008: METCRAX 2006: Meteorological experiments in Arizona's MeteorCrater. Bull. Amer. Meteor. Soc., 89, 1665-1680, https://doi.org/ 10.1175/2008BAMS2574.1.

_ , S. W. Hoch, J. D. Horel, and A. Charland, 2014: Relationship between particulate air pollution and meteorological variables in Utah's Salt Lake Valley. Atmos. Environ., 94, 742-753, https://doi.org/10.1016/j.atmosenv.2014.06.012.

Wiersema, D. J., K. A. Lundquist, and F. K. Chow, 2020: Mesoscale to microscale simulations over complex terrain with the immersed boundary method in the Weather Research and Forecasting Model. Mon. Wea. Rev., 148, 577-595, https:// doi.org/10.1175/MWR-D-19-0071.1.

Wilczak, J. M., and Coauthors, 2019: The Second Wind Forecast Improvement Project (WFIP2): Observational field campaign. Bull. Amer. Meteor. Soc., 100, 1701-1723, https://doi.org/ 10.1175/BAMS-D-18-0035.1.

Zängl, G., 2002: An improved method for computing horizontal diffusion in a sigma-coordinate model and its application to simulations over mountainous topography. Mon. Wea. Rev., 130, 1423-1432, https://doi.org/10.1175/1520-0493(2002) $130<1423$ :AIMFCH $>2.0 . \mathrm{CO} ; 2$.

- 2012: Extending the numerical stability limit of terrain-following coordinate models over steep slopes. Mon. Wea. Rev., 140, 37223733, https://doi.org/10.1175/MWR-D-12-00049.1.

Zardi, D., and C. D. Whiteman, 2013: Diurnal mountain wind systems. Mountain Weather Research and Forecasting: Recent Progress and Current Challenges, F. K. Chow et al., Eds., Springer, 35-119.

Zhong, S., C. D. Whiteman, X. Bian, W. J. Shaw, and J. M. Hubbe, 2001: Meteorological processes affecting the evolution of a wintertime cold air pool in the Columbia basin. Mon. Wea. Rev., 129, 2600-2613, https://doi.org/10.1175/1520-0493(2001) 129<2600:MPATEO >2.0.CO;2. 